Mancini, Lorenzo. Estrategias de desarrollo en torno a los recursos naturales: una revisión crítica de la literatura.

\title{
ESTRATEGIAS DE DESARROLLO EN TORNO A LOS RECURSOS NATURALES: UNA REVISIÓN CRÍTICA DE LA LITERATURA
}

\section{DEVELOPMENT STRATEGIES AND NATURAL RESOURCES: A CRITICAL REVIEW OF THE LITERATURE}

\author{
LORENZO MANCINI \\ Universidad Complutense De Madrid \\ mancio.27@libero.it
}

Fecha de recepción: noviembre de 2012

Fecha de aceptación: mayo de 2013

\begin{abstract}
RESUMEN
Desde los albores de la ciencia económica, los recursos naturales han estado al centro del análisis de los académicos. En los últimos años, el auge del sector de extracción ha dado un renovado impulso al debate en torno a las implicaciones económicas de tales recursos, al tiempo que han proliferado una serie de iniciativas vinculadas con ese sector. El presente artículo estudia críticamente las principales aportaciones de la literatura científica acerca de la relación entre recursos naturales y estrategias de desarrollo. El propósito es identificar y sistematizar aquellas condiciones que pueden promover un proceso de desarrollo económico, entendido como cambio estructural, a partir de la explotación de tales recursos.
\end{abstract}

PALABRAS CLAVE: recursos naturales; maldición de los recursos; enfermedad holandesa; desarrollo económico; industrialización; eslabonamientos

\section{ABSTRACT}

From the dawn of the economic science, natural resources have been at the centre of the analysis by scholars. During last years, the boom of the extractive sector has given a renewed momentum to the debate about economic implications of those recourses, while a series of initiatives linked with that sector has been spreading. This paper critically studies the main contributions of the cientific literature on the relation between natural resources and development strategies. The objective is to identify and systematize those conditions that could promote an economic development process, defined as a structural change, based on the exploitation of thos resources.

KEY WORDS: natural resources; resource curse; Dutch Disease; economic development; industrialization; linkages

JEL: O1; O2; O3

\section{INTRODUCCIÓN}

El potencial de los recursos naturales para conformar la estructura económica, social y política de los países ha sido una cuestión ampliamente debatida a lo largo del 
Mancini, Lorenzo. Estrategias de desarrollo en torno a los recursos naturales: una revisión crítica de la literatura.

tiempo. En algunos casos han tenido un papel relevante para el desarrollo económico, mientras que en otros su contribución ha sido marginal, llamando la atención de los académicos. Recientemente, el interés en el tema ha resurgido con más vigor, debido al auge de las materias primas y a la proliferación de nuevas iniciativas económicas en los países en desarrollo vinculadas con la explotación de las mismas.

Considerando la hipótesis de que en torno a los recursos naturales se puede definir una estrategia de desarrollo económico entendido como cambio estructural, la pregunta central de la presente investigación es la siguiente: ¿qué condiciones pueden promover una industrialización a partir de tales recursos? ${ }^{1}$

Identificando y estudiando críticamente las principales aportaciones de autores que analizan tales condiciones, desde diversos enfoques, el propósito del presente trabajo es contribuir al debate actual sobre las nuevas estrategias de desarrollo sistematizando los diferentes elementos interpretativos en un esquema de análisis. Ello permitirá tener una herramienta de estudio y valoración aplicable a tales iniciativas.

Los académicos suelen abordar la cuestión a través del análisis de un conjunto de hipótesis, variables y mecanismos amplio y diferenciado. Considerando imprescindible el estudio de las distintas posturas sostenidas, se ha optado por una metodología de investigación basada en la revisión crítica de la literatura. Sin embargo, ésta resulta extensa y dispersa entre numerosas vertientes, incluso dentro de una línea de pensamiento; en consecuencia, la selección de referencias, parte fundamental de ese estudio, ha sido elaborada con la intención de extraer, de los planteamientos generales de cada enfoque, los elementos esencialmente relevantes a la pregunta central de investigación.

El trabajo está organizado de la siguiente manera. En la primera sección, se estudian algunas de las contribuciones consideradas seminales con referencia a la relación entre recursos naturales y desarrollo económico. Se dedica una atención específica al fenómeno de la "enfermedad holandesa" (Dutch Disease).

En la segunda, se examinan críticamente las contribuciones de matriz institucionalista, destacando las vertientes argumentativas atribuibles a la postura conocida como la maldición de los recursos naturales (resource curse).

La tercera sección revisa la literatura que enriquece la orientación institucionalista con elementos históricos y estructuralistas y con aportaciones de otra derivación.

La parte final recoge las diferentes interpretaciones, identificando las condiciones que responden a la pregunta de investigación. Se analizan críticamente las principales coincidencias, divergencias e incompatibilidades entre las mismas. El

\footnotetext{
${ }^{1}$ La definición de recursos naturales adoptada en el presente trabajo incluye materias primas de extracción (minerales, petróleo, gas natural y otros hidrocarburos) y productos agrícolas. Por industrialización se entiende un proceso por el cual, a lo largo de un cierto horizonte temporal, el sector manufacturero cobra mayor relevancia económica en la estructura productiva de un determinado país. Consideramos tal proceso como una parte integral de otro fenómeno más amplio, el desarrollo económico, adoptando la línea interpretativa sugerida por la UNIDO (2009: 4).
} 
Mancini, Lorenzo. Estrategias de desarrollo en torno a los recursos naturales: una revisión crítica de la literatura.

apéndice contiene una tabla resumen de lo anterior. Por último, se discuten las limitaciones del presente trabajo y las posibles líneas de investigación adicional.

\section{RECURSOS NATURALES, DESARROLLO Y EL FENÓMENO DE LA "ENFERMEDAD HOLANDESA"}

El estudio de la relación entre recursos naturales y desarrollo ha despertado un significativo interés desde los albores de la disciplina económica. Entre las primeras contribuciones que dedicaron un esfuerzo particular a la comprensión de ese nexo, es posible destacar las atribuibles a la teoría del producto primario (staple thesis). Se argumenta que un impulso inicial procedente de las exportaciones de materias primas es capaz de estimular el crecimiento y la diversificación en otras áreas productivas, a través de la mayor atracción de capitales y empleo (Watkins, 1963) 2 .

En los años cincuenta y sesenta del siglo pasado, unos académicos empezaron a mirar con moderado optimismo el potencial de tales recursos (Lewis, 1958; Viner, 1966). Algunos argumentaron que tal dotación podía en parte contribuir a un general despegue de la economía hacia etapas superiores de industrialización (Rostow, 1964). Sin embargo, surgieron también interpretaciones más escépticas que destacaron principalmente los siguientes aspectos: el deterioro secular de los términos de intercambio reales de los productos primarios con respecto a los manufacturados (Prebisch, 1950; Singer, 1950), las elevadas fluctuaciones de precios de las materias primas en el mercado internacional (Nurkse, 1953) y las dificultades para generar eslabonamientos en las actividades primarias (Hirschman, 1961).

Sucesivamente, a partir de la observación de un evento concreto, tomó vigor un debate en torno al fenómeno conocido como "enfermedad holandesa". Según la interpretación generalmente sostenida, un incremento fuerte y sostenido de los ingresos procedentes de la exportación de recursos naturales de un país es capaz de afectar, vía apreciación del tipo de cambio real, la competitividad externa de otros sectores transables. Por ende, a través de una serie de mecanismos directos e indirectos, podría generarse una desindustrialización de la economía en cuestión (Corden y Neary, 1982; Krugman, 1987).

Según el modelo teórico al cual se hace referencia general, la desindustrialización puede ocurrir a través de los dos mecanismos siguientes. En primer lugar, directamente, debido a que el empleo, la producción y otros recursos de la economía empiezan a moverse y orientarse al sector energético (a menos que el mismo sea un enclave puro), al considerar que este tiene un mayor producto marginal (efecto movimiento de recursos o resource movement effect). Al mismo tiempo, el incremento de ingresos en la economía, además de apreciar el tipo de cambio nominal, puede impulsar una mayor demanda de productos transables y no transables (efecto consumo o consumption effect). Asumiendo que el precio de los primeros es determinado en el mercado internacional (el modelo general se basa en una economía de pequeño tamaño), ese aumento de la demanda se traduce en parte en mayores importaciones. Así, los precios en el sector no transable aumentan, así como los costes de los inputs y los salarios. Todo ello reduce los

\footnotetext{
${ }^{2}$ Véase: Innis, H., 1930. The fur trade in Canada: an introduction to Canadian economic history (Toronto, 1930; $2^{\mathrm{a}}$ ed., 1956), citado en: Watkins (1963).
} 
Mancini, Lorenzo. Estrategias de desarrollo en torno a los recursos naturales: una revisión crítica de la literatura.

beneficios de las empresas en el sector transable no energético y afecta la competitividad de sus exportaciones.

En segundo lugar, el desplazamiento (crowding out) de la industria puede manifestarse indirectamente, a través del incremento relativo de los salarios respecto a la productividad del trabajo (y por ende del coste unitario laboral) como consecuencia de la mayor demanda de empleo generada por la expansión del sector energético.

Algunos académicos destacan, además de los mecanismos anteriores, el proceso siguiente: asumiendo la hipótesis de que la manufactura tiene un elevado potencial para estimular la productividad y el nivel de cualificación de los recursos humanos a través de determinados procesos de aprendizaje (learning by doing), la "enfermedad holandesa" debilitaría un sector clave para el crecimiento económico. Se argumenta que la explotación de recursos naturales, siendo en general una actividad asociada a un bajo contenido tecnológico y a un empleo de trabajo con nivel de cualificación relativamente escaso, estimularía una acumulación de capital humano limitada (Kim, 1998; Gylfason et al., 1999; Bravo-Ortega y De Gregorio, 2005). Bajo el supuesto de que los trabajadores pueden trasladarse hacia el sector de extracción (atraídos por un auge del mismo), se sostiene que se genera un consiguiente desplazamiento de ciertas actividades de formación y capacitación, causando así una erosión progresiva del capital humano presente en la economía. Una prolongada desindustrialización pues podría afectar la trayectoria de algunos procesos de aprendizaje y por ende el crecimiento económico. Sachs y Warner (2001) concentran la atención, además, sobre el posible desplazamiento de la iniciativa empresarial: durante el auge, las inversiones privadas se orientan más al sector transable intensivo en recursos naturales.

La literatura que se ha desarrollado en torno a la "enfermedad holandesa" coincide en adoptar un enfoque predominantemente macroeconómico. Las condiciones necesarias de las estrategias propuestas se identifican en políticas monetarias, fiscales y cambiarias prudentes (Budina, Pang y Wijnbergen, 2006; Gianella, 2007; Baunsgaard et al., 2012). La mayoría de los académicos, respaldados por cierta evidencia empírica, reconocen a la volatilidad de precios de las materias primas como un problema central, matizando su repercusión sobre el flujo de los ingresos estatales y sobre las decisiones de inversión productiva. Con el propósito de contrastar tal factor adverso, argumentan a favor de la orientación de los ingresos generados por los recursos naturales a la creación de fondos de reserva con funciones específicas (aunque existen divergencias sobre en qué condiciones deberían basarse) ${ }^{3}$. Ello en nuestra opinión es una importante aportación de tal literatura.

\footnotetext{
${ }^{3}$ Con frecuencia, se sugiere crear un fondo con función de mera esterilización de los ingresos fiscales (acumulando reservas en divisas) y otro fondo orientado a asegurar el bienestar social de las generaciones futuras (permitiendo la inversión de los ingresos recaudados). Para enfrentar la volatilidad, autores afines al Banco Mundial (Sinnott et al., 2010) proponen también la negociación de instrumentos financieros de cobertura. Cabe evidenciar que una parte sustancial de la literatura publicada hasta hoy sobre los fondos de estabilización gira en torno a las pocas experiencias prácticas existentes. Además, hay todavía escasos estudios que investigan el tema en relación a los efectos del auge de materias primas más reciente. Al respecto, véase: Kutan y Wyzan (2005).
} 
Mancini, Lorenzo. Estrategias de desarrollo en torno a los recursos naturales: una revisión crítica de la literatura.

De un análisis crítico de los modelos teóricos más destacados acerca de la "enfermedad holandesa", es posible afirmar que, aunque ellos mantengan en general una lógica argumental consistente, la mayoría de las conclusiones que presentan derivan de hipótesis iniciales muy estrictas (Corden y Neary, 1982). Principalmente se evidencian las siguientes: el equilibrio de pleno empleo, la perfecta movilidad de los factores productivos, la estabilidad de la tecnología y la asunción de modelos muy simplificados de la economía. Los académicos han respondido a algunas limitaciones incorporando asunciones más sofisticadas y considerando un papel más activo de la intervención macroeconómica (Bruno y Sachs, 1982: 26-29; Corden, 1984: 374-376; van Wijnbergen, 1984). Por otro lado, los trabajos empíricos han ido investigando con más atención los mecanismos sugeridos por la teoría, cuestionando algunos resultados (Davis, 1995; Sarraf y Jiwanji, 2001; Ismail, 2010).

Cabe considerar que no parece posible evidenciar un consenso generalizado y comparable sobre cómo interactúan en concreto todos los mecanismos que afectan la industrialización supuestos en los modelos de la "enfermedad holandesa". Ello es debido a la dificultad de dirimir un debate cuya discordancia reside en referencias teórico-interpretativas divergentes y en el uso de metodologías de investigación distintas (Algieri, 2011). Emerge un decidido esfuerzo de los académicos en averiguar si un país tiene o no los síntomas del fenómeno, pero sin lograr comprender plenamente a través de qué canales específicos se concretan y porqué se produce en unos casos sí y en otros no. La literatura prefiere hacer hincapié en los adversos efectos cambiarios y en el crecimiento, pero sin llegar a una evidencia empírica conclusiva acerca del impacto de la "enfermedad" sobre la industrialización (Magud y Sosa, 2010). Además, resalta con frecuencia una clara influencia del pensamiento neoclásico, en cuanto que los resultados económicos están determinados básicamente por los mecanismos del mercado, pero sin conceder mucho espacio al análisis de las instituciones y de otras perspectivas.

Otra limitación de la literatura analizada es la escasa comprensión de las implicaciones de la "enfermedad holandesa" para las economías en fases incipientes de industrialización. Ello es debido a la carente profundidad de análisis sobre las características estructurales específicas de los países estudiados. Así la débil competitividad del sector transable manufacturero puede estar relacionada no sólo con una apreciación cambiaria (que no siempre ha ocurrido tras un boom energético), sino también con deficiencias estructurales internas de la economía. Puede revelarse útil estudiar el grado de influencia que el sector manufacturero doméstico puede ejercer en el mecanismo de distribución de la renta generada por los recursos naturales. En el caso de que tal industria tenga una elevada capacidad de apropiarse de parte de la misma renta, tal hecho actuaría como mecanismo de atenuación a la "enfermedad holandesa" (es decir: los ingresos que logra capturar la industria pueden ser destinados a defender su propia competitividad). Además, cabe considerar que países distintos pueden tener una composición interna de su industria manufacturera diferente, cuyas ramas, a su vez, responderían de manera peculiar a una expansión del sector intensivo en recursos naturales.

Por último, en la literatura estudiada, no emerge una evidencia robusta de que la explotación de tales recursos se caracteriza por un bajo nivel tecnológico per se, ni que siempre se asocia a una reducción del gasto público en educación. Parte de las 
Mancini, Lorenzo. Estrategias de desarrollo en torno a los recursos naturales: una revisión crítica de la literatura.

argumentaciones analizadas contradicen la experiencia de algunos países, cuyas inversiones en capital humano a partir del sector primario, permitieron impulsar una industrialización todavía incipiente (Wright y Czelusta, 2002). Parece por tanto importante indagar otras contribuciones sobre nuestro tema que puedan aportar algunas consideraciones históricas, estructurales y sobre el entorno institucional.

\section{LAS CONTRIBUCIONES INSTITUCIONALISTAS Y LA MALDICIÓN DE LOS RECURSOS NATURALES}

En las últimas décadas, han proliferado una serie de contribuciones que constituyen el cuerpo de una amplia literatura acerca del fenómeno conocido como la maldición de los recursos. La mayoría de tales trabajos suelen hacer referencia a la labor seminal de Sachs y Warner $(1995 ; 2001)$ los cuales, examinando los datos relativos a un grupo de países considerados ricos en recursos naturales, han mostrado la evidencia de que, en general, tal abundancia se ha correlacionado negativamente con el crecimiento económico ${ }^{4}$. En torno a este resultado, se han desarrollado numerosos trabajos de investigación que hacen hincapié en una fundamentación teórica del crecimiento básicamente neoclásica y en una metodología que privilegia la modelización econométrica.

Al respecto, cabe precisar que un estudio crítico de tal literatura requiere tener presente que la Economía Ambiental se ha construido, en cierta medida, a partir de un enfoque neoclásico ${ }^{5}$. Por ello, ha tendido a heredar las mismas limitaciones de la Economía del Bienestar pigouviana, entre las cuales estaría la abstracción del entorno institucional. La reciente consolidación del neoinstitucionalismo ha aportado a la teoría económica un marco analítico integral para la consideración del papel de las instituciones en la gestión de los recursos naturales ${ }^{6}$.

Una vertiente interpretativa atribuible a la postura de la maldición ha incorporado tales elementos teóricos neoinstitucionalistas a una visión neoclásica de la economía. En línea general, esa literatura argumenta que un significativo incremento de ingresos generado por los recursos naturales fomentaría actividades de búsqueda de posiciones rentistas (rent-seeking), debilitando las relaciones institucionales y productivas, y afectando indirectamente al crecimiento económico (Auty, 2001) $)^{7}$.

La interpretación sobre el rentismo hereda elementos neoclásicos y de la teoría de la elección pública (public choice): la inadecuada gobernanza de los recursos es atribuida principalmente a fallos del Estado y de ciertas políticas gubernamentales,

\footnotetext{
${ }^{4}$ Para un análisis crítico más detallado sobre el tema, véanse: Lederman y Maloney (2002; 2008) y Stevens (2003).

${ }^{5}$ Por Economía Ambiental consideramos la subdiscpilina de la ciencia económica que hace referencia, entre otras, al estudio de dos grandes cuestiones: las externalidades y la asignación intergeneracional óptima de recursos agotables.

${ }^{6}$ La literatura considerada en el presente trabajo suele referirse a las instituciones en sentido amplio, tal y como es propuesto por North (1990: 3).

${ }^{7}$ Algunos trabajos (en su mayoría empíricos y cuyo análisis supera el propósito del presente trabajo) han dado origen, además, a una interpretación de la maldición que asocia los recursos naturales a conflictos, guerras civiles, inestabilidad política y autoritarismo estatal (Collier y Hoeffler, 2004; Ross, 2004).
} 
Mancini, Lorenzo. Estrategias de desarrollo en torno a los recursos naturales: una revisión crítica de la literatura.

así como a la acción colectiva de grupos de interés influyentes ${ }^{8}$. Frecuentemente, los recursos naturales se conciben como una mera dotación, en una economía esencialmente modelizada y abstracta, donde los líderes políticos tienen comportamientos determinísticos, racionales y de índole predatoria. Con tales asunciones, esa postura no sólo atribuye un rol marginal a toda política industrial a partir de los recursos naturales, sino que parece incompleta, al pasar por alto el papel y la estructura de las fuerzas sociales y políticas.

Algunos académicos que podemos considerar en línea con la más reciente interpretación institucionalista del Banco Mundial (Canuto y Giugale, 2010; Sinnott et al., 2010), atribuyen la explicación de la maldición a la estructura de gobernanza de los recursos naturales y a la calidad de las instituciones (Mehlum et al., 2006; Robinson et al., 2006). En base a ello, consideran prioritarias las condiciones de estabilidad macroeconómica, la democracia y la transparencia en la gestión de las rentas. El concepto de calidad institucional es recogido también por autores afines a la visión del Fondo Monetario Internacional (FMI), qué añaden la apertura comercial entre los elementos favorecedores de toda estrategia económica sobre los recursos naturales (Arezki y van der Ploeg, 2007) ${ }^{9}$.

Estudiando la literatura, no emerge una evidencia empírica ampliamente compartida y conclusiva sobre los resultados de una maldición causada por un débil entorno institucional. Una limitación de esa argumentación deriva de las divergencias tanto conceptuales como en la medición de los recursos naturales y de la calidad de las instituciones (Brunnschweiler, 2008) ${ }^{10}$. A menudo, se exponen semi-tautologías basadas en correlaciones de variables (a veces espurias), sin considerar la existencia de una causación circular ente la debilidad institucional y los recursos naturales (además de incurrir en problemas de endogeneidad econométrica). Muchos estudios empíricos al respecto tienden a concentrarse en conjuntos heterogéneos de países, acudiendo a datos de sección cruzada, sin recoger las características estructurales específicas y resultando además sensibles al período de tiempo observado ${ }^{11}$.

\footnotetext{
${ }^{8}$ Se conjetura con frecuencia que el fuerte flujo de renta asociado a los recursos naturales permite al Estado recaudar un ingreso tal que le concede un margen para reducir su necesidad de ejercer la presión fiscal general y obtener así un cierto consenso político. Ello crearía las condiciones para comportamientos rentistas por parte del Gobierno, fomentando en la economía relaciones paternalistas y clientelares (patronage), hasta fenómenos de corrupción en torno a la pugna distributiva por la renta (Leite y Weidmann, 1999). Al respecto, véanse: Tornell y Lane (1999); Torvik (2002); Hammond (2011).

${ }^{9}$ La idea subyacente de tal propuesta es que la apertura comercial estimularía la exposición a la competencia de mercado y a una mayor posibilidad de transferencia tecnológica: ambas consideradas como vías al crecimiento económico con mejor calidad institucional. Tal postura, sin embargo, parece criticable porque pasa por alto, por ejemplo, los efectos del escalonamiento tarifario en recursos naturales aplicado hoy en los países más industrializados.

${ }^{10}$ Para una visión crítica sobre las divergencias conceptuales, véanse: Stijns (2001); Lederman y Maloney (2002; 2008); Isham et al. (2003); Sala-i-Martin y Subramanian (2003).

11 En la literatura estudiada es posible encontrar afirmaciones que se arriesgan por asemejarse a meras tautologías cuando consideran que la riqueza en recursos naturales, en presencia de instituciones débiles, afectaría el desempeño económico; tal asunción es a menudo utilizada para suportar argumentaciones que consideran la calidad institucional como una variable clave (Sinnott et al., 2010). Por otro lado, emerge un esfuerzo creciente de los académicos en el uso de variables instrumentales en los modelos econométricos para afrontar el problema de endogeneidad asociado a la cuestión institucional (Arezki y van der Ploeg, 2007).
}

Papeles de Europa

Vol. 26, Núm. 2 (2013): 33-63

http://dx.doi.org/10.5209/rev_PADE.2013.v26.n2.44183 
Mancini, Lorenzo. Estrategias de desarrollo en torno a los recursos naturales: una revisión crítica de la literatura.

Más importante, se percibe una cierta tendencia a asumir a priori que los recursos naturales son propiedad del Estado, sin profundizar en las características específicas de cada experiencia histórica observada ${ }^{12}$. Tal postura deja en un plano secundario no sólo el análisis del complejo proceso de acción colectiva que tiene lugar sobre los comunes, sino también aspectos institucionales importantes como la estructura de los derechos de propiedad, el control y el marco legal. Consideraciones que parece necesario tener en cuenta en una estrategia de industrialización, ya que afectan a la actividad productiva asociada con los recursos naturales (Ahrend, 2005; Gaddy e Ickes, 2005; Luong y Weinthal, 2010).

En el tema de la propiedad, heredando elementos del pensamiento neoliberal, una parte de los académicos considera que el sector de extracción es más eficiente cuando está en manos privadas (Sinnott et al., 2010). Pero, sin embargo, no se encuentra una evidencia empírica persuasiva al respecto (Hertog, 2010).

En las contribuciones analizadas, emerge una cierta pretensión de definir un modelo de buenas instituciones aplicables a todas las economías asumiendo, de manera simplista, que las que han sido positivas para unas, serían apropiadas para otras. Tal generalización no permite identificar qué condiciones institucionales caracterizaron la experiencia histórica de países ricos en recursos naturales que, a partir de ellos, impulsaron una industrialización todavía incipiente (Findlay y Lundhal, 1999; Feinstein, 2005; Blomström y Kokko, 2007). ${ }^{13}$ La pura interpretación institucionalista neoclásica parece ahistórica e inadecuada a contestar a nuestra pregunta de investigación respecto al contexto específico de las economías en desarrollo.

Otra carencia relevante de la literatura analizada es la falta de percepción de que la conformación de las instituciones puede derivar como resultado de la recíproca interacción de aquellas con las características estructurales de una economía y con las decisiones microeconómicas empresariales, a su vez asociadas a la dinámica del mercado internacional de los recursos naturales. Este último aspecto sugiere tener en cuenta con más detenimiento la posición específica de las economías periféricas respecto a la economía global y el papel de la internacionalización de la producción. Por último, debido a que se tiende a considerar el entorno institucional con abstracción de las condiciones históricas y estructurales, la interpretación puramente institucionalista neoclásica no permite comprender cómo gestionar las rentas fiscales, con fines de diversificación productiva según las características y las necesidades específicas de las economías en fases incipientes de industrialización. Para concluir, parece preciso indagar la incorporación de otros enfoques explicativos que complementen la literatura anteriormente analizada con otros elementos interpretativos.

\footnotetext{
${ }^{12}$ Los estudios empíricos suelen analizar los datos agregados por países y en períodos históricos en los cuales se mezclan experiencias distintas en el régimen de propiedad y control, público y privado, sobre el sector de extracción.

${ }^{13}$ Wright y Czelusta (2002) aportan cierta evidencia de cómo los recursos naturales han podido ser aprovechados como base a partir de la cual fue posible desarrollar distintas ramas de la manufactura, además de promover importantes avances en el progreso tecnológico. También Feinstein (2005: 174-175) considera que, a través de una política industrial activa, la minería permitió aumentar la demanda de bienes de equipo, impulsando la transformación industrial de Sudáfrica en los años cincuenta y sesenta.
} 
Mancini, Lorenzo. Estrategias de desarrollo en torno a los recursos naturales: una revisión crítica de la literatura.

\section{RECURSOS NATURALES Y ESTRATEGIA DE DESARROLLO: OTRAS INTERPRETACIONES CERCANAS A LA INSTITUCIONALISTA}

\subsection{Elementos históricos y estructurales}

En las últimas décadas, a partir sobre todo de las aportaciones de autores cepalinos, una creciente literatura se ha interesado en estudiar una estrategia de desarrollo económico a partir de los recursos naturales, incorporando elementos estructuralistas a una fundamentación teórica institucionalista. Algunos académicos han contribuido con la aportación de una cierta perspectiva histórica, según ellos considerada necesaria para explicar el impacto de la herencia pasada sobre la estructura productiva de una economía y, además, para entender el cambio institucional asociado al proceso de desarrollo económico.

Estas interpretaciones superan, en nuestra opinión, a las neoinstitucionalistas neoclásicas estudiadas anteriormente gracias al especial hincapié que hacen en la interrelación existente entre las condiciones históricas y estructurales (a menudo descuidadas en la literatura empírica atribuible a la maldición de los recursos) y la evolución de las instituciones, en el marco de una visión centro-periferia.

La interpretación estructuralista más genuina considera que la estructura productiva de una economía influye de manera determinante sobre el entorno institucional: la dinámica del cambio estructural se asocia a la modificación o incorporación de las "reglas del juego" (Di Filippo, 2009). Tal visión se acompaña a la consideración del impacto de la herencia histórica sobre la estructura social, haciendo emerger el papel de aquellos grupos de interés que logran controlar los recursos naturales e influir sobre el cambio institucional.

La estrategia de desarrollo sugerida hace hincapié en el concepto de aglomeración productiva o cluster (Porter, 1990). La hipótesis central es que una aglutinación similar en torno a la explotación de los recursos naturales, surgida en origen de manera espontánea por impulso de las fuerzas del mercado, es capaz en el largo plazo de lograr avances de productividad y de conducir a una diversificación hacia la manufactura (Ramos, 1999; CEPAL, 2005) ${ }^{14}$.

Incorporando el concepto de efecto de eslabonamiento o linkage (Hirschmann, 1961), esa literatura interpreta el cluster de recursos naturales como el "motor" a partir del cual se activan enlaces con la manufactura local. Se asume, además, que la aglomeración atraviesa idealmente una especie de ciclo de vida por etapas y que la intervención pública puede promover el proceso de maduración ${ }^{15}$. Con la idea de poder efectivamente acelerar tal tendencia (considerada natural), se argumenta en defensa de

\footnotetext{
14 La CEPAL (2005) distingue el concepto de aglomeración por pertenecer a un entorno institucional y geográfico específico; tal noción puede incluir otros conceptos, como los de cadena del valor y de red entre empresas. Se asume que la aglomeración de actividades en torno a los recursos naturales puede producir efectos complementarios de aprendizaje e innovación, a diferencia de lo que ocurriría según un mero enfoque conceptual de red empresarial. Evidenciamos que esa postura acude principalmente a una metodología basada en estudios de caso históricos y supera el formalismo neoclásico que caracteriza la literatura estudiada en los párrafos anteriores.

${ }^{15}$ En tal planteamiento, sin embargo, queda claro que todo ello no sigue un automatismo hacia un éxito cierto. Para Ramos (1999), un cluster maduro exitoso se caracteriza por mejoras continuas de la innovación tecnológica, apoyadas por políticas selectivas de fomento a la innovación.
} 
Mancini, Lorenzo. Estrategias de desarrollo en torno a los recursos naturales: una revisión crítica de la literatura.

una política industrial activa, pero que no renuncia a exponer las empresas a la lógica de la competencia del mercado y del comercio internacional, acompañando sus impulsos productivos.

Según la visión anterior, en una aglomeración la relación entre recursos naturales e industrialización pasa por la interacción entre las empresas, sus mercados y las instituciones (Buitelaar, 2001); además es necesaria una cierta masa crítica de actores para que se genere una dinámica virtuosa endógena y, al mismo tiempo, se plantea la hipótesis de que la presencia de fallos del mercado puede limitar la colaboración entre los agentes (CEPAL, 2005).

En síntesis, según esa visión, el entorno institucional es condicionado por la herencia histórica y las características estructurales. El Estado juega un rol fundamental en fomentar la aglomeración productiva y la coordinación entre todos, apoyando la generación de instituciones favorables para impulsar el conocimiento y la innovación tecnológica. Tal interpretación sin embargo no parece descuidar el plano microeconómico, considerando la colaboración público-privada auxiliar a la estrategia de desarrollo. Algunas contribuciones estructuralistas han sido influyentes sobre el pensamiento más reciente que hace hincapié en la dimensión productiva de tal estrategia que se analiza a continuación.

\subsection{La dimensión productiva}

En la literatura estructuralista estudiada, la industrialización se percibe como un factor de progreso técnico y como eje de la transformación productiva. Incorporando el concepto de sistema de innovación, se reconoce que los cambios que ocurren en los mercados globales y en el avance tecnológico requieren una cierta capacidad de innovación y adaptación local para poder fomentar el cambio estructural (Dos Santos y Tavares Dalcol, 2009) ${ }^{16}$. La adopción y la adecuación de tecnologías e innovaciones a partir de los recursos naturales son consideradas condiciones claves para escalar segmentos de mayor valor agregado en la cadena global ${ }^{17}$. Se confía en que una diversificación manufacturera basada en la innovación pueda impulsar los procesos de aprendizaje y formación de los recursos humanos (Peek et al., 2008; Teixeira y Ferraro, 2009; Morriss et al., 2011: 95). De tal manera, esta visión supera el supuesto neoclásico de tecnología estable y contrasta, además, con la hipótesis de desplazamiento del capital humano asociada al sector de extracción.

Las aportaciones históricas y estructuralistas han sido absorbidas por distintos académicos y organismos internacionales que hacen hincapié en la dimensión productiva, rechazando la hipótesis del sector de extracción como enclave. En general, hay coincidencia en reconocer que, a partir de los recursos naturales, pueden ser impulsados efectos de eslabonamiento industrial (Heum et al., 2003; Klueh et al., 2007; Teka, 2011). Tales mecanismos suelen diferenciar entre los que

\footnotetext{
${ }^{16}$ En general, el concepto de sistema de innovación es entendido como red de organizaciones (públicas y privadas), cuyas actividades crean y modifican nuevas tecnologías. El enfoque se centra en la innovación, qué puede basarse en procesos de aprendizaje a nivel sectorial o en una tecnología específica usada en varias industrias. Se conjetura que el sistema puede permitir crear y ampliar el conocimiento y la innovación en distintos sectores de la economía (Freeman, 1987).

${ }^{17}$ En tal interpretación, el concepto de cadena global del valor suele asociarse a una secuencia de actividades relacionadas y dependientes entre sí, necesarias para llevar un producto o un servicio desde la fase de concepción hasta su uso final.
} 
Mancini, Lorenzo. Estrategias de desarrollo en torno a los recursos naturales: una revisión crítica de la literatura.

se desarrollan hacia delante (forward o downstream), referidos al procesamiento, distribución y comercialización de las materias primas, y los que se desarrollan hacia atrás (backward o upstream), que incluyen el suministro de insumos, componentes manufacturados y bienes de equipo a la industria de extracción. Éstos últimos recogen el mayor interés de los académicos debido a que son considerados más intensivos en trabajo, elemento a su vez asociado a un cambio estructural (Aragón y Rud, 2011; Kaplinski et al., 2011; Oyejide y Adewuyi, 2011).

Hay amplio consenso en considerar esencial la colaboración público-privada en las actividades de Investigación y Desarrollo para fomentar la innovación local a partir de los recursos naturales. Se argumenta que existe un fuerte potencial para impulsar eslabonamientos laterales (sidestream), desde el sector de extracción hacia: servicios financieros, logística, transporte, comunicación, entre otros (Buitelaar, 2001; Machinea y Vera, 2007; UN-ECA, 2011). Se asume que el sector de recursos naturales puede generar derrames (spillovers) hacia la manufactura a través de inversiones en nuevas capacidades y competencias locales, a su vez suscetibles de ser aplicadas de nuevo con provecho en la actividad de extracción (Ritter, 2001; Sæther, Isaksen y Karlsen, 2011). Todo ello generaría nuevos eslabonamientos de migración lateral de tecnología genérica que, desarrollada inicialmente para servir la industria de extracción, pueda aplicarse sucesivamente en otros sectores (Walker y Jourdan, 2003). En nuestra opinión, el planteamiento anterior parece especialmente apropiado para definir una estrategia de desarrollo en economías con capacidades tecnológicas domésticas limitadas, aunque requiere todavía más evidencia empírica de sostenio.

Una creciente literatura reconoce amplio espacio a la política industrial de aglomeración en torno a los recursos naturales para fomentar la capacidad endógena a través de la diversificación productiva. Se hace hincapié en que cada proyecto de inversión en el sector de extracción tiene características específicas y requiere la aplicación de una tecnología particular. Ello impulsaría la continua formación de conocimiento y capacidades locales, estimulando el progreso técnico a partir de los recursos naturales. Se insiste en las implicaciones para la diversificación de la manufactura y la sofisticación de las exportaciones (Hausmann y Rigobon, 2002; Hausmann, Hwang y Rodrik, 2005; CEPAL, 2008; UNIDO, 2009). La cuestión atrae también el interés de académicos que adoptan el concepto de "espacio de producto" o product space (Hidalgo et al., 2007; Yifu Lin, 2012: 218) y de autores afines al FMI (Ismail, 2010), aunque se acude a fundamentaciones teóricas y metodológicas diferentes.

Hay un consenso emergente sobre la importancia de una política industrial activa que fomente las aglomeraciones productivas (CEPAL-GIZ, 2012; KSA, 2012; Sigam y Garcia, 2012; UNCTAD, 2012a; b) ${ }^{18}$. En primer lugar, las variables claves de la estrategia serían el suministro público de la infraestructura básica requerida por la explotación de los recursos naturales, los incentivos a la innovación tecnológica, el

\footnotetext{
${ }^{18}$ En el tercer Foro Global sobre Commodities (Global Commodities Forum; Ginebra, 23 y 24 de enero de 2012) de la UNCTAD se ha constatado que el reciente boom de los commodities, en general, no se ha traducido en una diversificación de la producción y de las exportaciones en los países en desarrollo que dependen más de ellas. Por ello, en el Foro citado, se ha defendido una política industrial más activa, en colaboración con el sector privado, para impulsar un mayor desarrollo manufacturero a partir de los clusters en actividades complementarias a las de la industria de extracción.
} 
Mancini, Lorenzo. Estrategias de desarrollo en torno a los recursos naturales: una revisión crítica de la literatura.

apoyo a la red de proveedores locales y las compras con preferente contenido local (UNCTAD, 2010; The World Bank, 2012).

En segundo lugar, destaca una fuerte coincidencia en argumentar que la inversión en la infraestructura física, tecnológica y de comunicación, suministrada para la explotación de los recursos naturales, puede fomentar los efectos de eslabonamiento industrial (Haglund, 2011; UN-ECA, 2011; VCC, 2011b; Sigam y Garcia, 2012; Yifu Lin, 2012: 31). Ese planteamiento tiene cierto respaldo empírico (Kuramoto, 2000; Morris, Kaplinsky y Kaplan, 2011; Oyejide y Adewuyi, 2011). Pérez (2010) enfatiza también el papel de las tecnologías de la información y las comunicaciones. Además, algunos matizan el potencial de desarrollo derivante de una infraestructura a escala regional y consideran importante una mayor cooperación supranacional (UN-ECA, 2004: 146; AUC, 2009: 8; VCC, 2011a; UNCTAD, 2012a).

En tercer lugar, emerge con evidencia la consideración de la inversión extranjera como elemento clave de la estrategia de desarrollo y como aspecto que merece un estudio específico en el tema de las industrias de extracción (Moran, 2011). Los académicos institucionalistas más puros tienden a estudiar el papel de tal inversión respecto a la generación de la renta fiscal, en estrecha relación con la cuestión de la trasparencia en la gestión de la misma. Por otro lado, los de orientación estructuralista enfocan mayormente el interés sobre las implicaciones en términos de demanda de empleo e inputs locales y sobre la potencial transferencia de tecnología desde las empresas foráneas (Kaplinsky et al., 2011; UNIDO, 2011). El marco regulador de la inversión extranjera es considerado un elemento estratégico para poder activar los eslabonamientos industriales y algunos recuperan el debate sobre los requisitos de desempeño a los inversores (UNCTAD, 2003; UN-ECA, 2011: 1134; VCC, 2011b) ${ }^{19}$. Parece relevante incorporar estas consideraciones debido al elevado grado de transnacionalización alcanzado por el sector de los recursos naturales y por las implicaciones que se derivan para las economías con capacidades tecnológicas limitadas (UNCTAD, 2007).

En cuarto lugar, las interpretaciones anteriores aportan un análisis del contexto geoeconómico y de la inserción externa en el mercado global como variables estratégicas en la relación recursos naturales-industrialización. Hay un creciente interés sobre los procesos de integración y de cooperación regional, con la hipótesis que el comercio y la inversión interregional (entre economías en similares fases incipientes de industrialización) pueden favorecer el desarrollo conjunto de tecnología y la diversificación exportadora (UN-ECA, 2004; CEPAL, 2008) ${ }^{20}$.

Por último, a diferencia de la literatura de la maldición, los enfoques estructuralistas destacan un creciente interés en estudiar la estructura de la propiedad y del control sobre los recursos naturales, el marco regulador de la inversión y las políticas de

\footnotetext{
${ }^{19}$ La UNCTAD (2003: 237-255) evidencia que el mecanismo de regulación formal de la inversión extranjera ha sido un instrumento ampliamente utilizado en los países escandinavos y de Oceanía, específicamente respecto a los recursos naturales y en una etapa de desarrollo en la cual las exportaciones primarias eran dominantes.

${ }^{20}$ Sin embargo, la cuestión encuentra divergencias también entre interpretaciones afines. Por ejemplo, Machinea y Vera (2007) concluyen que las exportaciones fueron un elemento dinámico en generar eslabonamientos productivos para algunos países ricos en recursos naturales. Por otro lado, Feinstein (2005) sostiene que, en el caso de Sudáfrica, las empresas mineras extranjeras empezaron a diversificar hacia la manufactura en parte como respuesta a la introducción de barreras comerciales a la importación.
} 
Mancini, Lorenzo. Estrategias de desarrollo en torno a los recursos naturales: una revisión crítica de la literatura.

contenido local (Klueh et al., 2007; Bain \& Company and Tozzini Freire Advogados, 2009; Likoski, 2009; Aragón y Rud, 2011; CEPAL-GIZ, 2012). Sin embargo, no emerge una evidencia clara y comparable sobre los efectos de éstas últimas iniciativas, debido a divergencias conceptuales y metodológicas entre los estudios. Todos los elementos señalados abren el espacio de condiciones que cabe tener en cuenta en la estrategia de desarrollo analizada en nuestro trabajo.

\section{CONCLUSIONES}

\subsection{Resultados}

La presente investigación ha intentado contribuir al debate actual en torno a las estrategias de desarrollo vinculadas a la explotación de recursos naturales, contestando a la pregunta sobre qué condiciones pueden promover una industrialización a partir de ellos. Como resultado de este trabajo, podemos resumir a continuación una serie de conclusiones (tabla 1 en anexo).

El estudio de la literatura ha permitido destacar también la presencia de significativas divergencias entre las interpretaciones. En primer lugar, los estructuralistas consideran necesaria pero no suficiente la condición de estabilidad macroeconómica. En ello, coinciden parcialmente con las recientes posturas afines al Banco Mundial. Sin embargo, estas últimas destacan por hacer hincapié en la transparencia de la gestión de la renta, la "buena" gobernanza de los recursos naturales y la calidad de las instituciones (Canuto y Giugale, 2010: 113; Sinnott et al., 2010) ${ }^{21}$. Siempre esta postura se separa de la estructuralista debido a que intenta validar algunos supuestos de la literatura de la maldición sobre el rentismo incorporando nuevos elementos de revisión de la estrategia propuesta.

En segundo lugar, desde la interpretación estructuralista se insiste en que la gobernanza y el fortalecimiento institucional son condiciones favorecedoras de la estrategia de desarrollo, pero sin la pretensión de definir un modelo universal de instituciones de buena calidad (CEPAL-GIZ, 2012) ${ }^{22}$. En coincidencia con la reciente visión afín al Banco Mundial, se mantiene el consenso sobre la idoneidad de la transparencia en la gestión de los ingresos generados por los recursos naturales, pero se pone el acento sobre la necesidad de que el Estado capte más renta, con progresividad, para reinvertirla con fines de diversificación productiva y cambio estructural (CEPAL, 2008: 60; CEPAL, 2012: 269) ${ }^{23}$.

En tercer lugar, los primeros modelos de la "enfermedad holandesa" y la postura de la maldición divergen de otras interpretaciones por tener una concepción mecanicista y economicista del proceso de industrialización, analizando la dinámica de las puras variables macroeconómicas. El libre funcionamiento del mercado y del sector privado son considerados esenciales para el crecimiento económico. Los estructuralistas y la

\footnotetext{
${ }^{21}$ Al respecto, la iniciativa de transparencia EITI (denominada así por su sigla inglés - Extractive Industries Transparency Initiative - y respaldada, entre otros, por el Banco Mundial) encuentra un consenso creciente en la literatura estudiada, siendo considerada como vía para responsabilizar los Gobiernos frente a los actores económicos (Keefer, 2003; UN-ECA, 2004; 2011; Haglund, 2011).

${ }^{22}$ Véase la reciente argumentación, procedente de la esfera cepalina, de institucionalizar los mecanismos para el manejo macroeconómico anticíclico frente a la volatilidad de las materias primas (Bárcena, 2012).

${ }^{23}$ En concreto, se hace hincapié sobre la destinación de tales ingresos a actividades de innovación tecnológica, aprendizaje y capacitación para fomentar la dotación de capital humano (CEPAL, 2012: 103).
}

Papeles de Europa

Vol. 26, Núm. 2 (2013): 33-63

http://dx.doi.org/10.5209/rev_PADE.2013.v26.n2.44183 
Mancini, Lorenzo. Estrategias de desarrollo en torno a los recursos naturales: una revisión crítica de la literatura.

interpretación afín al Banco Mundial en cambio abogan por una colaboración públicoprivada $^{24}$.

En cuarto lugar, los estructuralistas destacan por tener un acercamiento más propositivo y favorable a la política industrial. En ello coinciden con la más reciente visión afín al Banco Mundial en cuanto que ambas reconocen la cooperación entre Estado y sector privado como condición favorecedora de una estrategia de desarrollo que no se limita, además, al simple manejo macroeconómico. Sin embargo, con frecuencia la literatura sostiene genéricamente la necesidad de una cooperación público-privada, pero sin llegar a formular propuestas para concretarla. En nuestra opinión, para que esa pueda ser considerada una condición promotora de la estrategia de desarrollo, hay que contextualizarla en base a un estudio previo que permita comprender las características específicas de cada economía.

Adicionalmente, aunque la cuestión institucional es considerada un elemento estratégico en todas las interpretaciones analizadas, hay divergencias en la concreción de las condiciones necesarias. Los académicos de la "enfermedad holandesa" y de la postura de la maldición sostienen con fuerza la necesidad de que una economía tenga una cierta calidad institucional, pero sin concretar en qué consiste. La riqueza en recursos naturales es vista en asociación con el deterioro institucional por lo que estos académicos desconfían en el papel de la intervención gubernamental. En nuestra opinión, la gran aportación estructuralista es la consideración de que las instituciones pueden entenderse solo en relación con las características específicas de la estructura productiva. Estos últimos rechazan la idea de un modelo de "buenas" instituciones como condición indispensable para el desarrollo señalando, en cambio, la importancia de tener en cuenta la herencia histórica y las características estructurales específicas que influyen sobre las instituciones. Adquirir buenas instituciones de calidad requeriría por tanto un proceso de experimentación y adaptación en el tiempo. La política industrial y la gestión de las rentas fiscales como apoyo para cambiar la estructura productiva pueden ser instrumentos, adicionales y no únicos para modelar una ventaja comparativa dinámica (refutando así la idea de su estabilidad, que sería favorable a la especialización primaria) con el propósito de acelerar el proceso de industrialización a partir de (y no simplemente basado en) los recursos naturales.

Por último, la cuestión del entorno institucional es aceptada como prioritaria pero es muy debatida. Delante de la fuerte divergencia en establecer un modelo de instituciones exitoso aplicable a todas las economías, puede reconocerse un margen de flexibilidad en la definición de las "reglas del juego", pero siempre basado en una colaboración activa entre actores públicos y privados. El punto crítico del debate está sobre el papel que juegan estos dos sectores de la economía, generando interpretaciones distintas sobre el potencial de la política industrial frente al rol de los actores privados. En nuestra opinión, ambos lados contribuyen a la dinámica del proceso de industrialización y deben ser considerados conjuntamente. No pueden ignorarse las estrategias empresariales en la redefinición del marco regulador e

\footnotetext{
${ }^{24}$ Por un lado, los estructuralistas atraen de nuevo el debate al campo de los primeros académicos de la Economía del Desarrollo que se interesaron a los recursos naturales, introduciendo la perspectiva de las economías periféricas y la herencia histórico-estructural (aspectos descuidados en la literatura desarrollada a partir de la observación de la "enfermedad holandesa"). Por otro lado, se alejan de aquellos por conceder más espacio a la colaboración con el sector privado.
}

Papeles de Europa

Vol. 26, Núm. 2 (2013): 33-63

http://dx.doi.org/10.5209/rev_PADE.2013.v26.n2.44183 
Mancini, Lorenzo. Estrategias de desarrollo en torno a los recursos naturales: una revisión crítica de la literatura.

institucional. El tema de los derechos de propiedad y el control sobre los recursos naturales entre Estado y sector privado, parcialmente descuidado en la postura de la maldición, parece tener una importancia estratégica para promover la industrialización. Una aportación importante puede venir de la investigación de aspectos concretos del marco regulador y contractual de los recursos naturales (Luong y Weinthal, 2010; Tordo, Tracy y Alfaa, 2011).

Como otro resultado del presente trabajo, evidenciamos una fuerte contradicción en la interpretación institucionalista neoclásica en cuanto que defiende una liberalización de la inversión en el sector de extracción sin tener en cuenta las implicaciones de esa propuesta según las características históricas y estructurales de las economías. En nuestra opinión tal postura no tiene en cuenta, por ejemplo, cómo ha podido influir la herencia del pensamiento neoliberal sobre la definición de aquellas instituciones que gestionan la renta energética. En segundo lugar, la misma visión propone la liberalización comercial como condición favorecedora de la estrategia económica (Arezki y van der Ploeg, 2007). Sin embargo, una mayor apertura externa en el sector de extracción puede tener implicaciones contradictorias con las iniciativas de desarrollo adoptadas en las economías que presentan un patrón comercial primario-exportador y una inserción externa asimétrica en el mercado internacional.

Siempre en el tema institucional, algunos estructuralistas consideran el marco regulador de los recursos naturales como condición estratégica para favorecer la industrialización. Sin embargo, en nuestra opinión, la intervención gubernamental no puede concentrarse únicamente en ello, descuidando totalmente la lógica detrás de las decisiones empresariales y la dinámica del mercado de los recursos naturales. El papel protagónico de las empresas transnacionales en el sector de extracción implica estudiar la dinámica de la internacionalización de la producción en interrelación con las características estructurales de las economías receptoras. Considerar uno de estos elementos ceteris paribus, recomendando por ejemplo la adopción de un modelo de "buenas" instituciones o, al contrario, la redefinición del marco regulador como medida única, no parece permitir comprender la complejidad de las relaciones productivas que pueden promover la industrialización.

Por otro lado, del análisis crítico de la literatura emerge que existe una amplia coincidencia entre las distintas interpretaciones analizadas en considerar la estabilidad macroeconómica como condición necesaria en cuanto que, con cierto respaldo empírico, se reconoce como factor adverso la volatilidad de los ciclos de precios internacionales y de flujos de capital asociada a los recursos naturales. En nuestra opinión, ese elemento parece especialmente relevante como base de partida para la estrategia de industrialización. En concreto, el consenso más amplio está en favor de una política fiscal prudente, aunque la vertiente del rentismo estatal en la postura de la maldición hace hincapié en poner vínculos estrictos al gasto público y rechaza todo tipo de intervención restrictiva sobre los movimientos de capitales.

Para concluir, contestando de tal manera a la pregunta central de investigación, podemos señalar que hay cierta coincidencia en considerar favorecedoras de la estrategia de industrialización las siguientes condiciones que cabe tener en cuenta: la constitución de fondos de reserva con los ingresos fiscales procedentes de la explotación de recursos naturales (con fines de estabilización macroeconómica y de 
Mancini, Lorenzo. Estrategias de desarrollo en torno a los recursos naturales: una revisión crítica de la literatura.

inversión de largo plazo en beneficio de las generaciones futuras) ${ }^{25}$, la acumulación de capital humano (promoción de procesos de aprendizaje, formación y cualificación de los recursos humanos), la innovación técnica a partir de los recursos naturales (inversión en actividades de Investigación y Desarrollo), la colaboración públicoprivada, la transferencia de conocimiento y tecnología entre empresas extranjeras y domésticas, la aglomeración colaborativa entre todos los actores involucrados en el contexto geográfico próximo a la fuente de extracción, la inversión en infraestructura física y de comunicación de apoyo a la explotación de los recursos naturales. Por último, hay creciente consenso en señalar la relevancia de la cooperación en ámbito regional sobre comercio e inversión en recursos naturales; sin embargo, en nuestra opinión, es preferible considerar esta condición como secundaria, debido a la complejidad de los procesos de integración regional.

La presente investigación evidencia las siguientes incompatibilidades entre los diversos enfoques. La primera es parcial y consiste en que, mientras las interpretaciones atribuibles a la "enfermedad holandesa" y a la postura de la maldición se esfuerzan por investigar las condiciones que conectan los recursos naturales con el crecimiento económico-productivo (o mejor, con su pobre resultado), los estructuralistas no se "conforman" con el mero crecimiento y se preocupan de indagar los elementos favorecedores del más complejo proceso de cambio estructural a partir de los recursos naturales. Interpretando la industrialización como parte de un fenómeno más amplio, el desarrollo económico, es posible superar tal diversidad de visión incorporando la componente del crecimiento.

En segundo lugar, otra incompatibilidad se deriva de las distintas metodologías de investigación adoptadas: por ejemplo, la postura de la maldición privilegia la modelización econométrica. Para superar algunas incomprensiones, podríamos combinar la visión puramente macroeconómica (que caracteriza la literatura sobre la "enfermedad holandesa"), con la microeconómica propuesta en otras interpretaciones. Al respecto, algunos recientes estudios analizados en el presente trabajo parecen muy sugerentes porque incorporan la dinámica de la economía global con las decisiones de los actores que participan en una misma aglomeración productiva en torno a los recursos naturales. Sin embargo, las hipótesis del efecto multiplicador de esa aglutinación y de los efectos de eslabonamientos industriales, como argumentado por una parte de la literatura estructuralista, adolecen de la limitación metodológica relativa a la cuantificación empírica del fenómeno.

En nuestra opinión, para solucionar algunas de las divergencias analizadas y evitar la búsqueda de una "piedra filosofal" de condiciones aplicables siempre con éxito a toda economía, puede ser más explicativo acudir a un estudio caso por caso. Ello permitiría averiguar las implicaciones críticas de cada una de aquellas sobre la estrategia de desarrollo de una realidad económica específica; todo ello ayudaría a aclarar cómo las distintas condiciones identificadas en el presente trabajo interactúan entre ellas. En conclusión, parece necesario integrar la metodología cuantitativo-econométrica con la investigación cualitativa, indispensable para

\footnotetext{
${ }^{25}$ En nuestra opinión, la creación de tales fondos puede contribuir a estabilizar el gasto corriente y agregar financiamiento contracíclico, frente al factor adverso de la volatilidad. Además, puede servir a estabilizar el mercado cambiario, regulando la oferta de divisas, aunque sirva una estrecha coordinación entre los decisores de política fiscal y cambiaria.
}

Papeles de Europa

Vol. 26, Núm. 2 (2013): 33-63

http://dx.doi.org/10.5209/rev_PADE.2013.v26.n2.44183 
Mancini, Lorenzo. Estrategias de desarrollo en torno a los recursos naturales: una revisión crítica de la literatura.

comprender las características históricas y estructurales de una economía, el funcionamiento del entorno institucional específico y la dinámica de las decisiones microeconómicas de los actores involucrados en la explotación de los recursos naturales.

\subsection{Limitaciones y futura investigación}

Cabe señalar algunas limitaciones del presente artículo. Se reconoce no haber dedicado un espacio más amplio a las interpretaciones de Economía Ecológica, relevantes para estudiar una estrategia de desarrollo menos "productivista" de la aquí planteada. Debido a una elección explícita previa, además, no se han analizado en detalle las contribuciones que que se acercan al debate sobre recursos naturales e industrialización desde una perspectiva de género, así como las que matizan aspectos antropológicos de territorialidad indígena sobre las fuentes de extracción. ${ }^{26}$ La decisión del presente trabajo ha sido evitar la pretensión de un acercamiento holístico para concentrarse con más detenimiento sobre algunas destacadas interpretaciones directamente relacionadas con la pregunta de investigación.

Del análisis crítico han emergido crecientes propuestas de defensa de una política pública activa sobre los recursos naturales. Entre ellas, destacan las que consideran la dirección estratégica de las empresas de propiedad estatal y la promoción de la creación de valor local por vía legislativa. La literatura existente al respecto parece aún incompleta debido a que muchas propuestas gubernamentales han surgido sólo recientemente. Por ello, es preciso realizar más investigaciones para averiguar el potencial impacto de ellas sobre el proceso de industrialización y descubrir qué condiciones pueden promover una política de contenido local a partir de los recursos naturales.

Complementar instrumentos de investigación cuantitativos y cualitativos, recogiendo datos primarios sobre una aglomeración productiva concreta permitiría averiguar la dinámica y la interrelación de las variables y mecanismos debatidos en la literatura para aclarar caso por caso la relevancia de las condiciones consideradas favorecedoras de una industrialización a partir de los recursos naturales.

\footnotetext{
${ }^{26}$ Sobre la perspectiva de género, véanse: Eftimie, Heller y Strongman (2009); Ward et al. (2011); Eftimie et al. (2012). 
Mancini, Lorenzo. Estrategias de desarrollo en torno a los recursos naturales: una revisión crítica de la literatura.

\section{REFERENCIAS}

AUC (African Union Commission) (2009): Africa mining vision. [pdf], AUC, febrero de 2009. Disponible en: <http://www.web.ca/ emi/concertationgrandslacs/pdf/AfricaMining-Vision-2009.pdf> [Acceso 5 de mayo de 2012].

Ahrend, R. (2005): "Can Russia break the 'resource curse'?", Eurasian Geography and Economics, [e-journal] Vol. 46(8), diciembre de 2005, 584-609. Disponible a través de: base de datos EconLit [Acceso 12 de mayo de 2012].

Algieri, B. (2011): "The Dutch Disease: evidences from Russia", Economic Change Restructuring, [e-journal] Vol. 44(3), agosto de 2011, 243-277. Disponible a través de: base de datos EconLit [Acceso 12 de mayo de 2012].

Aragón, F.M. y Rud, J.P. (2011): "Natural resources and local communities: evidence from a Peruvian gold mine". [pdf], Publicación presentada en: Yale University. New Haven, CT: Yale Department of Economics. Abril de 2011. Disponible en: $<$ http://www.econ.yale.edu/conference/neudc11/papers/paper_274.pdf> [Acceso 16 de mayo de 2012].

Arezki, R. y van der Ploeg, F. (2007): "Can the natural resource curse be turned into a blessing? The role of trade policies and institutions". [pdf] Autorizado para la distribución por: Mansur, A. Washington, D.C.: International Monetary Fund (IMF), IMF Working Paper WP/07/55, Middle East and Central Asia Department, marzo de 2007. Disponible en: http://www.imf.org/external/pubs/ft/wp/2007/wp07 55.pdf [Acceso 8 de mayo de 2012].

Auty, R.M. (2001): "The political economy of resource-driven growth", European Economic Review, [e-journal] Vol. 45(4-6), mayo de 2001, 839-46. Disponible a través de: base de datos ScienceDirect [Acceso 12 de mayo de 2012].

Bain \& Company y Tozzini Freire Advogados (2009): "Relatório III - desenvolvimento da cadeia produtiva de petróleo e gás e investimentos em E\&P”. [pdf]. San Pablo (Brasil), 26 de junio de 2009 ( $1^{\text {a }}$ ed.). Disponible en: <http://www.bndes.gov.br/SiteBNDES/export/sites/default/bndes_pt/Galerias/Arq uivos/empresa/pesquisa/chamada1/Relat_III.pdf> [Acceso 10 de enero de 2012].

Bárcena, A. (2012): "Gobernanza de los recursos naturales en América Latina y el Caribe”. [pdf]. Presentación en el seminario internacional sobre: 'Gobernanza de los recursos naturales en América Latina y el Caribe', Santiago de Chile: CEPAL-GIZ, 24 de abril de 2012. Disponible en: <http://www.cepal.org/drni/noticias/noticias/9/46409/PresentacionSE.pdf> [Acceso 10 de junio de 2012].

Baunsgaard, T., Villafuerte, M., Poplawski-Ribeiro, M. y Richmond, R. (2012): "Fiscal frameworks for resource rich developing countries". [pdf]. Autorizado para la distribución por: Cottarelli, C.. Washington, D.C.: International Monetary Fund (IMF), IMF Staff Discussion Note SDN/12/04. Fiscal Affairs Department. 16 de mayo de 2012. Disponible en: <http://www.imf.org/external/pubs/ft/sdn/2012/sdn 1204.pdf> [Acceso 27 de agosto de 2012].

Blomström, M. y Kokko, A.F. (2007): "From natural resources to high-tech production: the evolution of industrial competitiveness in Sweden and Finland". In: Lederman y Maloney (2007), obra citada.

Bravo-Ortega, C. y De Gregorio, J. (2005): "The relative richness of the poor? Natural resources, human capital and economic growth". [pdf], Policy Research Working Paper Series 3484, 1 de enero de 2005. The International Bank for Reconstruction and Development/The World Bank, Washington, D.C. Disponible en: <http://wwwwds.worldbank.org/external/default/WDSContentServer/WDSP/IB 
Mancini, Lorenzo. Estrategias de desarrollo en torno a los recursos naturales: una revisión crítica de la literatura.

/2005/02/16/000112742_20050216123529/Rendered/PDF/wps3484.pdf> [Acce_ so 13 de mayo de 2012].

Bruno, M. y Sachs, J. (1982): "Energy and resource allocation: a dynamic model of the 'Dutch Disease'”. [pdf], NBER Working Paper n. 852, febrero de 1982. Cambridge, MA: National Bureau of Economic Research (NBER). Disponible en: $<$ http://www.nber.org/papers/w0852.pdf?new_window=1> [Acceso 10 de mayo de 2012].

Brunnschweiler, C.N. (2008): "Cursing the blessings? Natural resource abundance, institutions, and economic growth". World Development, [e-journal] Vol. 36(3), marzo de 2008, 399-419. Disponible a través de: base de datos ScienceDirect [Acceso 20 de mayo de 2012].

Budina, N., Pang, G. y van Wijnbergen, S. (2006): "Nigeria: Dutch disease or debt overhang? Diagnosing the past, lessons for the future". [pdf], 6 de octubre de 2006. Disponible en: <http://siteresources.worldbank.org/INTDEBTDEPT/

Resources/20061012_06.pdf> [Acceso 20 de mayo de 2012].

Buitelaar, R.M. (compilador) (2001): "Aglomeraciones mineras y desarrollo local en América Latina", CEPAL, Alfaomega. (1ª ed., noviembre de 2001). México, D.F.: Alfaomega grupo editor S.A. de C.V. Disponible en: <http://idlbnc.idrc.ca/dspace/bitstream/10625/30489/79/117066.pdf> [Acceso 17 de mayo de 2012].

Canuto, O. y Giugale, M. (Ed.s) (2010): "The day after tomorrow. A handbook on the future of economic policy in the developing world". [pdf], The International Bank for Reconstruction and Development/The World Bank, Washington, D.C. Disponible en: <http://siteresources.worldbank.org/EXTPREMNET/Resources/TDAT_Book.pdf> [Acceso 10 de mayo de 2012].

CEPAL (Comisión Económica para América Latina) (2012): Cambio estructural para la igualdad. Una visión integrada del desarrollo. Trigésimo cuarto período de sesiones de la CEPAL (San Salvador, 27-31 de agosto de 2012). Naciones Unidas, Santiago de Chile, julio de 2012 (original en español). Disponible en: <http://www.eclac.cl/pses34/noticias/documentosdetrabajo/4/47424/2012-SES-

34-Cambio_estructural.pdf> [Acceso 1 de septiembre de 2012].

(2008): "La transformación productiva 20 años después. Viejos problemas, nuevas oportunidades". Machinea, J.L. (coord.). Mayo de 2008. Diponible en: <http://www.eclac.cl/publicaciones/xml/7/33277/2008-117-SES.32-

Latransformacion-WEB_OK.pdf> [Acceso 4 de mayo de 2012].

- (2005): Aglomeraciones en torno a los recursos naturales en América Latina y el Caribe: Políticas de articulación y articulación de políticas. Naciones Unidas, Santiago de Chile, 2005.

CEPAL y GIZ (Deutsche Gesellschaft für Internationale Zusammenarbeit GmbH) (2012): "Seminario internacional sobre: 'Gobernanza de los recursos naturales en América Latina y el Caribe"', Resumen de los debates. [pdf], Santiago de Chile: CEPAL-GIZ, 24-25 de abril de 2012. Disponible en: <http://www.cepal.org/drni/noticias/noticias/9/46409/ResumenDebates.pdf> [Acceso 10 de junio de 2012].

Collier, P., y Hoeffler, A. (2004): "Greed and grievance in civil war", Oxford Economic Papers, [e-journal] 56(4), octubre de 2004, 563-595. Disponible a través de: base de datos EconLit [Acceso 12 de mayo de 2012]. 
Mancini, Lorenzo. Estrategias de desarrollo en torno a los recursos naturales: una revisión crítica de la literatura.

Corden, W.M. (1984): "Booming sector and Dutch disease economics: survey and consolidation", Oxford Economic Papers. New series, [e-journal] 36(3), noviembre de 1984, 359-380. Disponible a través de: base de datos JSTOR [Acceso 12 de mayo de 2012].

Corden, W.M. y Neary, J.P. (1982): "Booming sector and de-industrialisation in a small open economy", The Economic Journal, [e-journal] Vol. 92(368), diciembre de 1982, 825-848. Blackwell Publishing. Disponible a través de: base de datos EconLit [Acceso 12 de mayo de 2012].

Davis, G.A. (1995): "Learning to love the Dutch disease: evidence from the mineral economies". World Development, [e-journal] Vol. 23(10), octubre de 1995, 17651779. Disponible a través de: base de datos ScienceDirect [Acceso 10 de mayo de 2012].

Di Filippo, A. (2009): "Estructuralismo latinoamericano y teoría económica". Revista CEPAL, [e-journal] n.98, agosto de 2009, 181-202. Santiago de Chile: CEPAL. Disponible en: <http://www.cepal.org/publicaciones/xml/4/36814/RVE98DiFilippo.pdf> [Acceso 10 de julio de 2012].

Dos Santos, S.B. y Tavares Dalcol, P.R. (2009): "Geographical proximity and innovation: evidences from the Campos Basin oil \& gas industrial agglomerationBrazil", Technovation, [e-journal] Vol. 29(8), agosto de 2009, 546-561. Disponible a través de: base de datos ScienceDirect [Acceso 15 de febrero de 2012].

Eftimie, A., Heller, K., Strongman, J., Hinton, J., Lahiri-Dutt, K., Mutemeri, N., con: Insouvanh, C., Godet Sambo, M. y Wagner, S. (2012): "Gender dimensions of Artisanal and Small-Scale Mining. A rapid assessment toolkit". [pdf], The International Bank for Reconstruction and Development/The World Bank, Washington, D.C. Disponible en: $<$ http://siteresources.worldbank.org/INTEXTINDWOM/Resources/Gender_and_A SM_Toolkit.pdf> [Acceso 5 de junio de 2012].

Eftimie, A., Heller, K. y Strongman, J. (2009): "Gender dimensions of the extractive industries: mining for equity". [pdf], Extractive Industries and Development Series n.8, agosto de 2009. The International Bank for Reconstruction and Development/The World Bank, Washington, D.C. Disponible en: $<$ http://siteresources.worldbank.org/EXTOGMC/Resources/eifd8_gender_equity. pdf> [Acceso 5 de junio de 2012].

Feinstein, C.H. (2005): An economic history of South Africa: conquest, discrimination and development. Cambridge University Press, Cambridge.

Findlay, R. y Lundahl, M. (1999): "Resource-led growth. A long-term perspective. The relevance of the 1870-1914 experience for today's developing economies". [pdf], Working Paper n.162, julio de 1999. The United Nations University; WIDER (World Institute for Development Economics Research). Disponible en: <http://www.wider.unu.edu/publications/working-papers/previous/en_GB/wp162/_files/82530858917962442/default/wp162.pdf> [Acceso 21 de mayo de 2012].

Freeman, C. (1987): Technology policy and economic performance: lessons from Japan. Pinter, Londres.

Gaddy, C. y Ickes, B. (2005): "Resource rents and the Russian economy", Eurasian Geography and Economics, [e-journal] Vol. 46(8), 2005, 559-583. Disponible en: $<$ http://www.brookings.edu/views/articles/gaddy/20051101.pdf> [Acceso 14 de abril de 2012]. 
Mancini, Lorenzo. Estrategias de desarrollo en torno a los recursos naturales: una revisión crítica de la literatura.

Gianella, C. (2007): "A golden rule for Russia? How a rule-based fiscal policy can allow a smooth adjustment to the new terms of trade". [pdf], OECD (Organisation for Economic Co-operation and Development) Economics Department Working Papers n.53715, enero de 2007. OECD Publishing. Disponible en: $<$ http://www.oecd-ilibrary.org/docserver/download/fulltext/514w02nctq6b.pdf? expires $=1340043905 \&$ id $=$ id\&accname $=$ guest $\&$ checksum =6BE33AE86975A90F8 8B73F86D6EE107B > [Acceso 8 de marzo de 2012].

Gylfason, T. (2001): "Lessons from the Dutch disease: causes, treatment, and cures". [pdf]. Para: STATOIL-ECON, volumen de la conferencia: The Paradox of Plenty, 22 de marzo de 2001. Disponible en: <https://notendur.hi.is/gylfason/pdf/ statoil22.pdf $>$ [Acceso 24 de abril de 2012].

Gylfason, T., Herbertsson, T.T. y Zoega, G. (1999): "A mixed blessing, natural resources and economic growth", Macroeconomic Dynamics, [e-journal] Vol. 3, 1999, 204-225. Disponible en: <https://notendur.hi.is/gylfason/pdf/ mixedblessing2.pdf $>$ [Acceso 10 de abril de 2012].

Haglund, D. (2011): "Blessing or curse? The rise of mineral dependence among lowand middle-income countries". [pdf]. Oxford, U.K.: Oxford Policy Management, diciembre de 2011. Disponible en: <http://www.opml.co.uk/sites/opml/files/ OPM\%20Blessing\%20or\%20curse.pdf> [Acceso 10 de mayo de 2012].

Hammond, J.L. (2011): "The resource curse and oil revenues in Angola and Venezuela", Science and Society, [e-journal] Vol. 75(3), julio de 2011, 348-78. Disponible en: <http://guilfordjournals.com/doi/pdf/10.1521/siso.2011.75.3.348> [Acceso 23 de abril de 2012].

Hausmann, R., Hwang, J. y Rodrik, D. (2005): "What you export matters". [pdf], NBER Working Paper n.11905, Cambridge, MA: National Bureau of Economic Research (NBER), diciembre de 2005, revisión de marzo de 2006. Disponible en: <http://www.nber.org/papers/w11905.pdf?new_window=1> [Acceso 23 de abril de 2012].

Hausmann, R. y Rigobon, R. (2002): "An alternative interpretation of the 'resource curse': Theory and policy implications". [pdf], NBER Working Paper n.9424, Cambridge, MA: National Bureau of Economic Research (NBER), diciembre de 2002. Disponible en: <http://www.nber.org/papers/w9424.pdf?new_window=1> [Acceso 15 de abril de 2012].

Hertog, S. (2010): "Defying the resource curse explaining successful state-owned enterprises in rentier states", World Politics, [e-journal] Vol. 62(2), abril de 2010, 261-301. Cambridge University Press. Disponible en: http://www.relooney.info/SI_Governance/Governance-Economic-Growth_17.pdf [Acceso 12 de abril de 2012].

Heum, P., Quale, C., Karlsen, J.E., Kragha, M. y Osahon, G. (2003): "Enhancement of local content in the upstream oil and gas industry in Nigeria. A comprehensive and viable policy approach". [pdf]. SNF Report n. 25/03; estudio conjunto: SNF Institute for Research in Economics and Business Administration (Bergen); RF Rogaland Research (Stavanger); K\&A - Kragha \& Associates (Lagos). Bergen, Stavanger, Lagos, agosto de 2003. Disponible en: <https://www.brazilfocus.com/App_Data/elib/SNF\%20Nigeria\%20Report\%202005.pdf> [Acceso 15 de febrero de 2012].

Hidalgo, C.A., Klinger, B., Barabási, A.-L. y Hausmann, R. (2007): "The product space conditions the development of nations", Science, [e-journal] Vol. 317(5837), 27 de julio de 2007, 482-487. Disponible en: <http://0- 
Mancini, Lorenzo. Estrategias de desarrollo en torno a los recursos naturales: una revisión crítica de la literatura.

www.sciencemag.org.cisne.sim.ucm.es/content/317/5837/482.full.pdf> [Acceso 10 de mayo de 2012].

Hirschman, A.O. (1961): La estrategia del desarrollo económico. Fondo de Cultura Económica, México, D.F. (1 $1^{\mathrm{a}}$ ed. en español, agosto de 1961). [Título original: The Strategy of Economic Development. New Haven; London: Yale University Press].

Isham, J., Pritchett, L., Woolcock, M. y Busby, G. (2003): "The varieties of the resource experience: how natural resource export structures affect the political economy of economic growth". [pdf], Middlebury College Discussion Paper n. 2003-08, Middlebury, Vermont: Middlebury College, Department of Economics. Disponible en: <http://papers.ssrn.com/sol3/papers.cfm?abstract_id=410364> [Acceso 2 de mayo de 2012].

Ismail, K. (2010): "The structural manifestation of the "Dutch disease": the case of oil exporting countries". [pdf]. Autorizado para la distribución por: Ulric Erickson von Allmen. Washington, D.C.: International Monetary Fund (IMF), IMF Working Paper WP/10/103, Strategy, Policy, and Review Department, abril de 2010. Disponible en: <http://www.imf.org/external/pubs/ft/wp/2010/wp10103.pdf> [Acceso 19 de junio de 2012].

Kaplinsky, R., Morris, M. y Kaplan, D. (2011): "A conceptual overview to understand commodities, linkages and industrial development in Africa". [pdf], Africa Export Import Bank. Disponible en: <http://oro.open.ac.uk/30534/2/2E4B0FCA.pdf> [Acceso 9 de mayo de 2012].

Keefer, P. (2003): "What does political economy tell us about economic development-and vice versa?", Annual Review of Political Science, [e-journal] Vol. 7, junio de 2004, 247-272. The International Bank for Reconstruction and Development/The World Bank, Development Research Group, Washington, D.C. Disponible en: <http://dss.ucsd.edu/ mnaoi/page4/POLI227/files/page1_9.pdf> [Acceso 4 de mayo de 2012].

Kim, Y.J. (1998): "Resource curse, overcommitment, and human capital", Journal of Economic Development, [e-journal] Vol. 23(2), diciembre de 1998, 103-121. Disponible en: <http://www.jed.or.kr/full-text/23-2/kim.PDF> [Acceso 16 de mayo de 2012].

Klueh, U., Pastor, G., Segura, A. y Zarate, W. (2007): "Inter-sectoral linkages and local content in extractive industries and beyond - the case of São Tomé and Príncipe". [pdf], Washington, D.C.: International Monetary Fund (IMF), IMF Working Paper WP/07/213, IMF African Department, septiembre de 2007. Disponible en: <http://www.imf.org/external/pubs/ft/wp/2007/wp07213.pdf> [Acceso 15 de febrero de 2012].

Krugman, P. (1987): "The narrow moving band, the Dutch disease and the competitive consequences of Mrs. Thatcher on trade in the presence of dynamic scale economies", Journal of Development Economics, [e-journal] Vol. 27(1-2), octubre de 1987, 41-55. Disponible a través de: base de datos ScienceDirect [Acceso 12 de mayo de 2012].

KSA (Kingdom of Saudi Arabia) (2012): "National Industrial Clusters Development Program". [pdf], Kingdom of Saudi Arabia, Ministry of Commerce \& Industry. Japanese-Saudi business opportunities forum. Tokio, Japón, 1 de febrero de 2012. Disponible en: <http://www.saudiarabia-jccme.jp/forumpdf/3-3.pdf> [Acceso 15 de julio de 2012].

Kuramoto, J.R. (2000): "Las aglomeraciones productivas alrededor de la minería: el caso de la Minera Yanacocha S.A." [pdf]. CEPAL, Serie Desarrollo Productivo 
Mancini, Lorenzo. Estrategias de desarrollo en torno a los recursos naturales: una revisión crítica de la literatura.

n.67, enero de 2000. Santiago de Chile: CEPAL, División de Desarrollo Productivo y Empresarial. Disponible en: <http://www.eclac.org/publicaciones/ $\mathrm{xml} / 7 / 4637 / \mathrm{lcl} 1312$ e.pdf $>$ [Acceso 12 de abril de 2012].

Kutan, A.M. y Wyzan, M.L. (2005): "Explaining the real exchange rate in Kazakhstan, 1996-2003: is Kazakhstan vulnerable to the Dutch disease?", Economic Systems, [e-journal] Vol. 29(2), junio de 2005, 242-255. Disponible a través de: base de datos ScienceDirect [Acceso 12 de mayo de2012].

Lederman, D. y Maloney, W.F. (2008): "In search of the missing resource curse". [pdf], Policy Research Working Paper 4766, noviembre de 2008, The World Bank Development Research Group Trade Team \& Latin America and the Caribbean Region Office of the Chief Economist. Disponible en: <http://wwwwds.worldbank.org/servlet/WDSContentServer/WDSP/IB/2008/11/04/000158349 20081104085814/Rendered/PDF/WPS4766.pdf> [Acceso 23 de mayo de 2012].

(2007): Natural resources, neither curse nor destiny. Palo Alto, CA: Stanford Economics and Finance; The International Bank for Reconstruction and Development/The World Bank, Washington, D.C.

- (2002): "Open questions about the link between natural resources and economic growth: Sachs and Warner revisited". [pdf], Central Bank of Chile Working Paper n.141, febrero de 2002. Disponible en: <http://www.bcentral.cl/eng/studies/ working-papers/pdf/dtbc141.pdf> [Acceso 25 de abril de 2012].

Leite, C. y Weidmann, J. (1999): "Does mother nature corrupt? Natural resources, corruption, and economic growth". [pdf], IMF Working Paper WP/99/85, julio de 1999, Washington, D.C.: International Monetary Fund (IMF). Disponible en: <http://www.imf.org/external/pubs/ft/wp/1999/wp9985.pdf> [Acceso 20 de abril de 2012].

Lewis, W.A. (1955): The theory of economic growth. R.D. Irwin, Homewood, IL, 1955. Likosky, M. (2009): "Contracting and regulatory issues in the oil and gas and metallic minerals industries", Transnational Corporations, [e-journal] Vol. 18(1), abril de 2009, 1-42. UNCTAD (United Nations Conference on Trade and Development), Division on Investment and Enterprise, Naciones Unidas, Nueva York y Ginebra. Disponible en: <http://archive.unctad.org/en/docs/diaeiia20097a1_en.pdf> [Acceso 12 de enero de 2012].

Luong, J. y Weinthal, E. (2010): Oil is not a curse: ownership structure and institutions in Soviet successor states. Cambridge studies in comparative politics. Cambridge University Press, Nueva York.

Machinea, J.L. y Vera, C. (2007): "Inserción internacional y políticas de desarrollo productivo". [pdf]. In: Machinea, J.L. y Serra, N., (Ed.s), 2007. Visiones del desarrollo en América Latina. Capítulo 10, 341-405. Santiago de Chile: CEPAL; Barcelona: Fundación CIDOB. Disponible en: <http://www.oei.es/salactsi/CIDOBCEPALVisiones.pdf> [Acceso 12 de mayo de 2012].

Maehle, N. (2012): "Boosting employment: lessons from natural resource abundant countries". [pdf]. Presentación en el seminario: 'Towards a new economic growth strategy for employment, decent work and development in Zambia'. GRZ/ILO/IMF National Conference. New Government Complex Lusaka, 21-22 de mayo de 2012. Disponible en: <http://www.imf.org/external/np/seminars/eng/ 2012/zambia/pdf/mae.pdf> [Acceso 2 de julio de 2012].

Magud, N. y Sosa, S. (2010): "When and why worry about real exchange rate appreciation? The missing link between Dutch Disease and growth". [pdf]. Autorizado para la distribución por: Robert Rennhack. Washington, D.C.: 
Mancini, Lorenzo. Estrategias de desarrollo en torno a los recursos naturales: una revisión crítica de la literatura.

International Monetary Fund (IMF), IMF Working Paper WP/10/271, Western Hemisphere Department, diciembre de 2010. Disponible en: <http://www.imf.org/external/pubs/ft/wp/2010/wp10271.pdf> [Acceso 19 de junio de 2012].

Manzano, O. y Rigobon, R. (2001): "Resource curse or debt overhang?" [pdf], NBER Working Paper n.8390, julio de 2001. National Bureau of Economic Research (NBER), Cambridge, MA. Disponible en: <http://www.nber.org/papers/w8390.pdf ?new_window $=1>$ [Acceso 12 de enero de 2012].

Mehlum, $\overline{\mathrm{H}}$., Moene, K. y Torvik, R. (2006): "Institutions and the resource curse", The Economic Journal, [e-journal] Vol. 116(508), enero de 2006, 1-20. Blackwell Publishing. Disponible a través de: base de datos JSTOR [Acceso 3 de mayo de 2012].

Moran, T.H. (2011): Foreign Direct Investment and development. Launching a second generation of policy research. Peterson Institute for International Economics, Washington, D.C., abril de 2011.

Morris, M., Kaplinsky, R. y Kaplan, D. (2011): "Commodities and linkages: industrialisation in Sub Saharan Africa". [pdf], MMCP (Making the Most of Commodities Programme) Discussion Paper n.13, octubre de 2011. Disponible en: <http://www.cssr.uct.ac.za/sites/cssr.uct.ac.za/files/pubs/MMCP\%20Paper\% 2013_0.pdf> [Acceso 10 de febrero de 2012].

North, D.C. (1990): Institutions, institutional change and economic performance. Political Economy of Institutions and Decisions collection. Cambridge University Press, Cambridge ( $1^{\text {a }}$ ed., 1990).

Nurkse, R. (1953): Problems of capital formation in underdeveloped countries. Oxford University Press, Oxford, 1953.

Oyejide, T.A. y Adewuyi, A.O. (2011): "Enhancing linkages of oil and gas industry in the Nigerian economy". [pdf], MMCP (Making the Most of Commodities Programme) Discussion Paper n.8, marzo de 2011. TPRTP (Trade Policy Research and Training Programme), Department of Economics, University of Ibadan. Disponible en: <http://commodities.open.ac.uk/8025750500453F86/\%28 httpAssets\%29/6D572F58F7E2A83B8025787E003A7258/\$file/Enhancing\%20lin kages\%20of\%20oil\%20and\%20gas\%20industry\%20in\%20the\%20Nigerian\%20e conomy.pdf> [Acceso 10 de febrero de 2012].

Peek, P., Fenard, J., Gantes, P. y Theiler, C. (2008): "Skills shortages in the global oil and gas industry. How to close the gap. Part l". [pdf], CRES (Centre de Recherches Entreprises et Sociétés). Diciembre de 2008. Disponible en: <http://www.cres.ch/Documents/SKILLS\%20SHORTAGE\%20PART\%201\%20pdf. pdf> [Acceso 12 de febrero de 2012].

Pérez, C. (2010): "Dinamismo tecnológico e inclusión social en América Latina: una estrategia de desarrollo productivo basada en los recursos naturales". Revista CEPAL, [e-journal] n.100, abril de 2010, 123-145. Santiago de Chile: CEPAL. Disponible en: <http://www.eclac.cl/publicaciones/xml/2/39122/RVE100Perez.pdf [Acceso 19 de mayo de 2012].

Porter, M.E. (1990): The competitive advantage of nations. The MacMillan, London, 1990.

Prebisch, R. (1950): "The economic development of Latin America and its principal problems". Republicado en: Economic Bulletin for Latin America, [e-journal] Vol. 7(1), 1962, 1-22. In:_Greenaway, D. y Morgan, C.W. (Ed.s) (1999): The economics of commodity markets. Elgar Reference Collection. International Library of Critical Writings in Economics, Vol. 105: 3-61. Cheltenham, U.K. y 
Mancini, Lorenzo. Estrategias de desarrollo en torno a los recursos naturales: una revisión crítica de la literatura.

Northampton, MA: Edward Elgar; distribuido por: American International Distribution Corporation, Williston, Vt.

Ramos, J. (1999): "Una estrategia de desarrollo a partir de los complejos productivos (clusters) en torno a los recursos naturales ¿una estrategia prometedora?" [pdf], Original: español. Naciones Unidas (CEPAL), Santiago de Chile, agosto de 1999. Disponible en: <http://www.eclac.cl/ddpeudit/proy/clusters/JRamos.pdf> [Acceso 10 de mayo de 2012].

Ritter, A.R.M. (2001): La aglomeración en torno a la minería en Canadá: estructura, evolución y funcionamiento. En: Buitelaar (2001): obra citada.

Robinson, J.A., Torvik, R. y Verdier, T. (2006): "Political foundations of the resource curse", Journal of Development Economics, [e-journal] Vol. 79(2), abril de 2006, 447-68. Disponible a través de: base de datos ScienceDirect [Acceso 4 de abril de 2012].

Ross, M. (2004): "What do we know about natural resources and civil war?", Journal of Peace Research, [e-journal] Vol. 41(3), mayo de 2004, 337-56. Disponible a través de: base de datos JSTOR [Acceso 15 de mayo de 2012].

Rostow, W.W. (1964): The stages of economic growth: a non-communist manifesto. Cambridge University Press, Cambridge, 1964.

Sachs, J.D. y Warner, A. (2001): "Natural resources and economic development: the curse of natural resources", European Economic Review, [e-journal] Vol. 45(4-6), mayo de 2001, 827-838. Disponible a través de: base de datos ScienceDirect [Acceso 15 de marzo de 2012].

(1995; revisado en 1997): "Natural resource abundance and economic growth". [pdf], NBER Working Paper n.5398, diciembre de 1995. Cambridge, MA: National Bureau of Economic Research (NBER). Disponible en: $<$ http://www.nber.org/papers/w5398.pdf?new_window=1> [Acceso 10 de abril de 2012].

Sæther, B., Isaksen, A. y Karlsen, A. (2011): "Innovation by co-evolution in natural resource industries: The Norwegian experience", Geoforum, [e-journal] Vol. 42(2011), 373-381. Disponible a través de: base de datos ScienceDirect [Acceso 8 de mayo de 2012].

Sala-i-Martin, X. y Subramanian, A. (2003): "Addressing the resource curse: an illustration from Nigeria". [pdf], NBER Working Paper n.9804, junio de 2003. National Bureau of Economic Research (NBER), Cambridge, MA. Disponible en: $<$ http://www.nber.org/papers/w9804.pdf?new_window=1> [Acceso 12 de mayo de 2012].

Sarraf, M. y Jiwanji, M. (2001): "Beating the resource curse: the case of Botswana". [pdf], The World Bank Environment Department Papers, Environmental Economics Series, octubre de 2001. The International Bank for Reconstruction and Development/The World Bank, Washington, D.C.:. Disponible en: $<$ http://www-

wds.worldbank.org/servlet/WDSContentServer/WDSP/IB/2002/09/24/000094946 02090504023362/Rendered/PDF/multiOpage.pdf> [Acceso 12 de mayo de 2012].

Sigam, C. y Garcia, L. (2012): "Extractive industries: optimizing value retention in host countries". [pdf]. Naciones Unidas (Ginebra: UNCTAD), Nueva York y Ginebra. Documento: UNCTAD/SUC/2012/1. Preparado en el marco de la conferencia: '15th African Oil, Gas and Minerals Trade and Finance Conference (OILGASMINE)', Congo 2012, y del evento especial: 'Natural Gas as an engine of growth', UNCTAD XIII, Qatar 2012. Disponible en: <http://unctadxiii.org/en/ 
Mancini, Lorenzo. Estrategias de desarrollo en torno a los recursos naturales: una revisión crítica de la literatura.

SessionDocument/suc2012d1_en.pdf> [Acceso 10 de junio de 2012].

Singer, H.W. (1950): "U.S. foreign investment in underdeveloped areas: the distribution of gains between investing and borrowing countries", The American Economic Review, [e-journal] Vol. 40(2), mayo de 1950, 473-85. Papers and proceedings of the sixty-second annual meeting of the American Economic Association. Disponible a través de: base de datos JSTOR [Acceso 12 de marzo de 2012].

Sinnott, E., Nash, J. y de la Torre, A. (2010): "Natural resources in Latin America and the Caribbean beyond booms and busts?" [pdf], The International Bank for Reconstruction and Development/The World Bank, Washington, D.C. Disponible en: <http://siteresources.worldbank.org/INTLAC/Resources/257803-1284336216 058/FlagshipReport.pdf> [Acceso 10 de abril de 2012].

Stevens, P. (2003): "Resource impact: a curse or a blessing". [pdf], Centre for Energy, Petroleum and Mineral Law and Policy, 25 de marzo de 2003, University of Dundee. Disponible en: <http://www.dundee.ac.uk/cepmlp/journal/html/Vol13/ article13-14.pdf> [Acceso 22 de mayo de 2012].

Stijns, J-P.C. (2005): "Natural resource abundance and economic growth revisited". [pdf], University of California, Berkeley Department of Economics, noviembre de 2000. Disponible en: $h$ ttp://are.berkeley.edu/fields/erep/seminar/f2000/stijns.pdf [Acceso 21 marzo de 2012].

Teixeira, F. y Ferraro, C. (2009): "Aglomeraciones productivas locales en Brasil, formación de recursos humanos y resultados de la experiencia CEPALSEBRAE". [pdf], CEPAL, Serie Desarrollo Productivo n.186, marzo de 2009. Santiago de Chile: CEPAL, Unidad de Desarrollo Industrial y Tecnológico. Avalaible at: <http://www.eclac.org/publicaciones/xml/5/35735/lcl3005e.pdf> [Acceso 3 de marzo de 2012].

Teka, Z. (2011): "Backward linkages in the manufacturing sector in the oil and gas value chain in Angola", [pdf], MMCP (Making the Most of Commodities Programme) Discussion Paper n.11, marzo de 2011. University of Cape Town y Open University. Disponible en: <http://www.cssr.uct.ac.za/sites/cssr.uct.ac.za/ files/pubs/MMCP\%20Paper\%2011_0.pdf> [Acceso 7 de enero de 2012].

The International Bank for Reconstruction and Development/The World Bank (2012): Increasing local procurement by the mining industry in West Africa. Road-test version. [pdf], The International Bank for Reconstruction and Development/The World Bank, Washington, D.C. Document of the World Bank, report n.66585AFR, enero de 2012. Disponible en: <http://siteresources.worldbank.org/INT ENERGY2/Resources/8411-West_Africa.pdf> [Acceso 27 de mayo de 2012].

Tordo, S., Tracy, B.S. y Arfaa, N. (2011): National Oil Companies and value creation. [pdf], World Bank working paper, Vol. 218(1), marzo de 2011 (versión anterior a la publicación); The International Bank for Reconstruction and Development/The World Bank, Washington, D.C. Disponible en: <http://siteresources.worldbank. org/INTOGMC/Resources/336099-1300396479288/noc_volume_I.pdf> [Acceso 5 de junio de 2012].

Tornell, A. y Lane, P.R. (1999): "The voracity effect". The American Economic Review, [e-journal] Vol. 89(1), marzo de 1999, 22-46. American Economic Association. Disponible a través de: base de datos JSTOR [Acceso 21 de marzo de 2012].

Torvik, R. (2002): "Natural resources, rent seeking and welfare". Journal of Development Economics, [e-journal] Vol. 67, 2002, 455-470. Disponible en: <http://www.svt.ntnu.no/iso/ragnar.torvik/jde.pdf> [Acceso 10 de mayo de 2012]. 
Mancini, Lorenzo. Estrategias de desarrollo en torno a los recursos naturales: una revisión crítica de la literatura.

UNCTAD (United Nations Conference on Trade And Development) (2012a): "Natural gas as an engine of growth". [pdf], evento especial UNCTAD XIII, Qatar National Convention Centre, Doha, 23 de abril de 2012. Sumario preparado por el Secretariado de la UNCTAD, 4 de mayo de 2012. Disponible en: <http://unctad.org/meetings/en/SessionalDocuments/td478_en.pdf>

[Acceso 16 de junio de 2012].

- (2012b): "Outcome of the Fifteenth African Oil, Gas and Minerals Trade and Finance Conference and Exhibition". [pdf], evento anterior a la conferencia UNCTAD XIII, 4-6 de abril de 2012, Brazzaville, Congo. Nota preparada por el Secretariado de la UNCTAD, 18 de abril de 2012. Disponible en: <http://unctad.org/meetings/en/SessionalDocuments/tdinf46_en.pdf>

[Acceso 16 de junio de 2012].

(2011): "Report of the Global Commodities Forum 2011". [pdf], The Second Global Commodities Forum. Palacio de Naciones, Ginebra, 31 de enero-1 de febrero de 2011. Document UNCTAD/SUC/2011/5. Disponible en: <http://unctad.org/en/docs/suc2011d5_en.pdf> [Acceso 3 de junio de 2012].

(2010): Creating business linkages: $\bar{a}$ policy perspective, [e-journal]. Naciones Unidas, Nueva York y Ginebra. Disponible en: <http://www.unctad.org>

[Acceso 15 de marzo de 2012].

(2007): World Investment Report 2007. Transnational corporations, extractive industries and development. [pdf], Naciones Unidas, Nueva York y Ginebra. Disponible en: <http://www.unctad.org/en/docs/wir2007_en.pdf> [Acceso 21 de marzo de 2012].

- (2003): "Foreign direct investment and performance requirements: new evidence from selected countries". [pdf], documento UNCTAD/ITE/IIA/2003/7. Octubre de 2003. Naciones Unidas, Nueva York y Ginebra. Disponible en: <http://unctad.org/en/docs/iteiia20037_en.pdf> [Acceso 6 de marzo de 2012].

UN-ECA (United Nations Economic Commission for Africa) (2011): "Minerals and Africa's development. The international study group report on Africa's mineral regimes". [pdf], UN-ECA, Adís Abeba (Etiopía). Noviembre de 2011. Disponible en: <http://www.africaminingvision.org/amv_resources/AMV/ISG\%20Report_eng. pdf> [Acceso 10 de junio de 2012].

- (2004): "Minerals cluster policy study in Africa. Pilot studies of South Africa and Mozambique". [pdf], UN-ECA, Adís Abeba (Etiopía). Diciembre de 2004. Disponible en: <http://www.uneca.org/eca_programmes/sdd/documents/mineral_cluster_study_ sa_mozambique.pdf $>$ [Acceso 27 de mayo de 2012].

UNIDO (United Nations Industrial Development Organization) (2011): "Commodities for industrial development: making linkages work". [pdf], UNIDO Working paper 01/2011. (Preparado por: Kaplinsky, R.), Development Policy and Practice, The Open University. Disponible en: <http://www.unido.org/fileadmin/user_media/ Services/Research_and_Statistics/statistics/WPMP\%2001\%20Commodities\%20 for\%20Industrial\%20Development\%20-\%20Maiking\%20Linkages\%20Work.pdf> [Acceso 12 de mayo de 2012].

- (2009): "Industrial Development Report 2009. Breaking in and moving up: new industrial challenges for the bottom billion and the middle-income countries". [pdf], Naciones Unidas, Ginebra. Disponible en: <http://www.unido.org/fileadmin/ user_media/Publications/IDR_2009_print.PDF> [Acceso 21 de mayo de 2012]. 
Mancini, Lorenzo. Estrategias de desarrollo en torno a los recursos naturales: una revisión crítica de la literatura.

Van Wijnbergen, S. (1984): "The Dutch disease: a disease after all?" Economic Journal, [e-journal] Vol. 94(373), marzo de 1984, 41-55. Disponible a través de: base de datos JSTOR [Acceso 3 de abril de 2012].

VCC (The Vale Columbia Center on Sustainable International Investment) (2011a): "Resource-based sustainable development in the lower Zambezi basin". [pdf], Columbia University, VCC, 1 de junio de 2011, borrador para la consultación. Disponible en: <http://www.vcc.columbia.edu/files/vale/content/Consultative Draft-_Zambezi_Resource_Based_Development_-_3_June_2011.pdf> [Acceso 4 de mayo de 2012$]$.

- (2011b): The Sixth Columbia International Investment Conference on: 'The Resource Boom and FDI in Africa: new players, new opportunities, a new agenda for development'. [pdf], Columbia University, Faculty House, Nueva York, 26-27 de octubre de 2011. Conference summary (preparado por: Jacky Mandelbaum). Disponible en: <http://www.vcc.columbia.edu/files/vale/content/ CIIC11_Summary_18_Nov_11.pdf> [Acceso 27 de mayo de 2012].

Viner, J. (1966): Comercio internacional y desarrollo económico. Obra en español. Colección de ciencias sociales, Serie de economía, Editorial Tecnos S.A., Madrid ( 2 a ed. 1966). [Título original: "International trade and economic development", $\left(1^{\mathrm{a}}\right.$ ed. $\left.)\right]$

Walker, M. y Jourdan, P. (2003): "Resource-based sustainable development: an alternative approach to industrialisation in South Africa". Minerals and Energy, [ejournal] Vol. 18(3), 25-43.

Ward, B., Strongman, J., Eftimie, A. y Heller, K. (2011): "Gender-sensitive approaches for the extractive industry in Peru. Improving the impact on women in poverty and their families: guide for improving practice". [pdf], Extractive Industries and Development Series n.24, diciembre de 2011. The International Bank for Reconstruction and Development/The World Bank, Washington, D.C. Disponible en: <http://siteresources.worldbank.org/INTOGMC/Resources/24web-REDS.pdf> [Acceso 5 de junio de 2012].

Watkins, M.H. (1963): "A Staple theory of economic growth". The Canadian Journal of Economics and Political Science, [e-journal] Vol. 29(2), mayo de 1963, 141158. Blackwell Publishing. Disponible a través de: base de datos JSTOR [Acceso 3 de marzo de 2012].

Wright, G. y Czelusta, J. (2002): "Exorcising the resource curse: minerals as a knowledge industry, past and present”. [pdf], Working Paper n.02008, julio de 2002. Stanford University Economics Department, Stanford, CA. Disponible en: <http://www-siepr.stanford.edu/workp/swp02008.pdf> [Acceso 21 de marzo de 2012].

Yifu Lin, J. (2012): New Structural Economics: a framework for rethinking development. [e-book], The International Bank for Reconstruction and Development/The World Bank, Washington, D.C. Disponible en: <http://siteresources.worldbank.org/DEC/Resources/84797-

1104785060319/598886-1104951889260/NSE-Book.pdf> [Acceso 5 de julio de 2012]. 
Mancini, Lorenzo. Estrategias de desarrollo en torno a los recursos naturales: una revisión crítica de la literatura.

\section{ANEXO}

Tabla 1. Condiciones para una estrategia de industrialización. Coincidencias, divergencias e incompatibilidades entre las interpretaciones analizadas.

\begin{tabular}{|c|c|c|c|c|}
\hline \multicolumn{5}{|c|}{ 1. Coincidencias y divergencias } \\
\hline $\begin{array}{l}\text { Condiciones para la } \\
\text { industrialización }\end{array}$ & “"Enfermedad holandesa” & $\begin{array}{c}\text { Institucionalista } \\
\text { neoclásica; postura de la } \\
\text { maldición }\end{array}$ & $\begin{array}{l}\text { Institucionalista- } \\
\text { estructuralista }\end{array}$ & $\begin{array}{l}\text { Institucionalista afín al } \\
\text { Banco Mundial y } \\
\text { revisionista de la } \\
\text { maldición }\end{array}$ \\
\hline Estabilidad macroeconómica & $\begin{array}{l}\text { Necesaria: anticíclica- } \\
\text { volatilidad. }\end{array}$ & $\begin{array}{l}\text { Necesaria: anticíclica- } \\
\text { volatilidad. }\end{array}$ & $\begin{array}{l}\text { Necesaria: anticíclica- } \\
\text { volatilidad. }\end{array}$ & $\begin{array}{l}\text { Necesaria: anticíclica- } \\
\text { volatilidad. }\end{array}$ \\
\hline Política cambiaria & $\begin{array}{l}\text { Evitar restricciones cambiarias } \\
\text { y controles al movimiento de } \\
\text { capitales. }\end{array}$ & $\begin{array}{l}\text { Evitar restricciones } \\
\text { cambiarias y controles al } \\
\text { movimiento de capitales. }\end{array}$ & $\begin{array}{l}\text { Parcial apoyo a controles al } \\
\text { movimiento de capitales a } \\
\text { corto plazo. }\end{array}$ & $\begin{array}{l}\text { Evitar restricciones } \\
\text { cambiarias y controles al } \\
\text { movimiento de capitales. }\end{array}$ \\
\hline Política monetaria & $\begin{array}{l}\text { No relevante bajo la hipótesis } \\
\text { de precios dados en el } \\
\text { mercado internacional. }\end{array}$ & $\begin{array}{l}\text { Relevante para esterilizar } \\
\text { los efectos de inflación. }\end{array}$ & $\begin{array}{l}\text { Relevante pero conflicto } \\
\text { (trilema imposible de política } \\
\text { económica). }\end{array}$ & $\begin{array}{l}\text { Relevante pero conflicto } \\
\text { (trilema imposible de política } \\
\text { económica). }\end{array}$ \\
\hline Política fiscal & $\begin{array}{l}\text { Prudente (restricciones } \\
\text { presupuestarias). }\end{array}$ & $\begin{array}{l}\text { Prudente (fondos de } \\
\text { estabilización; reglas y } \\
\text { restricciones } \\
\text { presupuestarias). }\end{array}$ & $\begin{array}{l}\text { Prudente (fondos de } \\
\text { estabilización y con fines de } \\
\text { desarrollo a largo plazo). }\end{array}$ & $\begin{array}{l}\text { Prudente (fondos de } \\
\text { estabilización y con fines de } \\
\text { desarrollo a largo plazo). }\end{array}$ \\
\hline
\end{tabular}


Mancini, Lorenzo. Estrategias de desarrollo en torno a los recursos naturales: una revisión crítica de la literatura.

\begin{tabular}{|c|c|c|c|c|}
\hline Política industrial & $\begin{array}{l}\text { Secundaria (posibles subsidios } \\
\text { fiscales al sector transable no } \\
\text { energético). }\end{array}$ & $\begin{array}{l}\text { Evitable por causa de la } \\
\text { debilidad institucional } \\
\text { estatal. }\end{array}$ & $\begin{array}{l}\text { Necesaria: política de } \\
\text { aglomeración y efectos de } \\
\text { eslabonamientos. }\end{array}$ & $\begin{array}{l}\text { Necesaria pero no } \\
\text { suficiente: posibles efectos } \\
\text { de eslabonamientos. }\end{array}$ \\
\hline $\begin{array}{l}\text { Acumulación de capital } \\
\text { humano y procesos de } \\
\text { aprendizaje }\end{array}$ & $\begin{array}{l}\text { Esencial para evitar el efecto } \\
\text { de desplazamiento. }\end{array}$ & $\begin{array}{l}\text { Esencial para evitar el } \\
\text { efecto de desplazamiento. }\end{array}$ & $\begin{array}{l}\text { Esencial para generar rentas } \\
\text { futuras basadas en } \\
\text { conocimiento. }\end{array}$ & $\begin{array}{l}\text { Esencial para generar } \\
\text { rentas futuras basadas en } \\
\text { conocimiento. }\end{array}$ \\
\hline Innovación tecnológica & $\begin{array}{l}\text { Hipótesis de tecnología } \\
\text { estable. }\end{array}$ & $\begin{array}{l}\text { Progreso técnico endógeno } \\
\text { basado en capital humano. }\end{array}$ & $\begin{array}{l}\text { Progreso técnico a partir de la } \\
\text { aglomeración productiva. }\end{array}$ & $\begin{array}{l}\text { Progreso técnico basado en } \\
\text { capital humano y } \\
\text { conocimiento. }\end{array}$ \\
\hline $\begin{array}{l}\text { Infraestructura física y de } \\
\text { comunicación }\end{array}$ & $\begin{array}{l}\text { No objeto de específica } \\
\text { atención. }\end{array}$ & $\begin{array}{l}\text { Importante pero riesgos } \\
\text { institucionales: prioridad al } \\
\text { sector privado y papel del } \\
\text { Estado como mero } \\
\text { suministrador de la } \\
\text { infraestructura básica. }\end{array}$ & $\begin{array}{l}\text { Importante para generar } \\
\text { efectos de eslabonamiento } \\
\text { industrial: requiere } \\
\text { colaboración público-privada. }\end{array}$ & $\begin{array}{l}\text { Importante para generar } \\
\text { efectos de eslabonamiento } \\
\text { industrial: requiere } \\
\text { colaboración público- } \\
\text { privada. }\end{array}$ \\
\hline Apertura comercial & $\begin{array}{l}\text { Modelos teóricos con } \\
\text { economías pequeñas y } \\
\text { cerradas. }\end{array}$ & $\begin{array}{l}\text { Necesaria para incorporar } \\
\text { tecnología y promover la } \\
\text { competencia de la } \\
\text { manufactura local. }\end{array}$ & $\begin{array}{l}\text { Riesgos de re-primarización } \\
\text { exportadora; posibilidad en } \\
\text { los procesos de integración } \\
\text { regional. }\end{array}$ & $\begin{array}{l}\text { Necesaria para incorporar } \\
\text { tecnología y promover la } \\
\text { competencia de la } \\
\text { manufactura local. }\end{array}$ \\
\hline Liberalización de la inversión & $\begin{array}{l}\text { Mantener la apertura a pesar } \\
\text { del fuerte ingreso de divisas. }\end{array}$ & $\begin{array}{l}\text { Esencial debido al papel } \\
\text { central del sector privado. }\end{array}$ & $\begin{array}{l}\text { Posibilidad de redefinir el } \\
\text { marco regulador-institucional } \\
\text { (renta fiscal y transferencia } \\
\text { de tecnología). }\end{array}$ & $\begin{array}{l}\text { Papel central de la inversión } \\
\text { privada, evitar un marco } \\
\text { regulador restrictivo a la } \\
\text { iniciativa empresarial, sobre } \\
\text { todo la extranjera. }\end{array}$ \\
\hline Cooperación regional & $\begin{array}{l}\text { No objeto de específica } \\
\text { atención. }\end{array}$ & $\begin{array}{l}\text { Afectada por la debilidad } \\
\text { institucional de algunos } \\
\text { Gobiernos. }\end{array}$ & $\begin{array}{l}\text { Posible espacio de } \\
\text { cooperación tecnológica e } \\
\text { infraestructural entre países } \\
\text { periféricos. }\end{array}$ & $\begin{array}{l}\text { Posible para difundir buenas } \\
\text { prácticas de transparencia } \\
\text { en la gestión de la renta } \\
\text { fiscal. }\end{array}$ \\
\hline
\end{tabular}

Papeles de Europa

Vol. 26, Núm. 2 (2013): 33-63 http://dx.doi.org/10.5209/rev_PADE.2013.v26.n2.44183 
Mancini, Lorenzo. Estrategias de desarrollo en torno a los recursos naturales: una revisión crítica de la literatura.

\begin{tabular}{|c|c|c|c|c|}
\hline \multicolumn{5}{|l|}{ 2. Incompatibilidades } \\
\hline $\begin{array}{l}\text { Enfoque prioritario de la } \\
\text { interpretación }\end{array}$ & $\begin{array}{l}\text { Crecimiento económico y } \\
\text { desindustrialización. }\end{array}$ & $\begin{array}{l}\text { Crecimiento económico } \\
\text { basado en recursos } \\
\text { naturales. }\end{array}$ & $\begin{array}{l}\text { Cambio estructural a partir de } \\
\text { los recursos naturales. }\end{array}$ & $\begin{array}{l}\text { Crecimiento económico } \\
\text { basado en recursos } \\
\text { naturales y objetivos } \\
\text { sociales. }\end{array}$ \\
\hline Instituciones & Limitadas o ausentes. & $\begin{array}{l}\text { Los recursos naturales se } \\
\text { asocian a instituciones } \\
\text { débiles. Es necesaria la } \\
\text { calidad institucional. }\end{array}$ & $\begin{array}{l}\text { Las características histórico- } \\
\text { estructurales determinan las } \\
\text { instituciones. Los } \\
\text { mecanismos institucionales } \\
\text { son la base relevante para la } \\
\text { política industrial. }\end{array}$ & $\begin{array}{l}\text { Los recursos naturales se } \\
\text { asocian a instituciones } \\
\text { débiles. Es necesaria una } \\
\text { buena gobernanza de los } \\
\text { recursos. }\end{array}$ \\
\hline Papel del Estado & $\begin{array}{l}\text { Limitada intervención con } \\
\text { subsidios fiscales. }\end{array}$ & $\begin{array}{l}\text { Rentismo estatal y debilidad } \\
\text { institucional. }\end{array}$ & $\begin{array}{l}\text { Esencial para promover una } \\
\text { política industrial de } \\
\text { aglomeración. }\end{array}$ & $\begin{array}{l}\text { Activo en la política } \\
\text { macroeconómica y en la } \\
\text { gobernanza de los recursos } \\
\text { naturales }\end{array}$ \\
\hline Sector privado & Esencial. & Esencial. & $\begin{array}{l}\text { Favorable a la colaboración } \\
\text { público-privada. }\end{array}$ & $\begin{array}{l}\text { Favorable a la colaboración } \\
\text { público-privada. }\end{array}$ \\
\hline $\begin{array}{l}\text { Metodología de investigación } \\
\text { principal }\end{array}$ & $\begin{array}{l}\text { Modelos teóricos y elevado } \\
\text { formalismo. }\end{array}$ & Modelos econométricos. & $\begin{array}{l}\text { Argumentación con datos, } \\
\text { explicación histórica, estudios } \\
\text { de caso. }\end{array}$ & $\begin{array}{l}\text { Modelos econométricos y } \\
\text { argumentación con datos. }\end{array}$ \\
\hline
\end{tabular}

Fuente: elaboración propia. 